\title{
An electron microscopic study of bacteriophages from marine waters
}

\author{
Hermann Frank \& Karlheinz Moebus \\ Max-Planck-Institut für Entwicklungsbiologie; \\ Spemannstr. 35/EM, D-7400 Tübingen, FRG \\ and \\ Biologische Anstalt Helgoland (Meeresstation); \\ $D$-2192 Helgoland, FRG
}

\begin{abstract}
The morphology of 75 bacteriophage strains isolated from water samples collected in the North Sea or in the northern Atlantic was studied by electron microscopy. Only tailed phages were observed (Bradley groups A, B, and C). According to structural similarities, the strains are ascribed to 12 groups, 5 of which comprise types of marine phages not reported before. Four of these 5 groups include phage types that have not been detected from any other source. Among the phages isolated from northern Atlantic water a high incidence was observed of strains the particles of which have long appendages. Certain types of the northern Atlantic phages investigated were derived only from samples collected either east or west of the Azores. This finding agrees with former observations pointing to the existence of different populations of closely related bacteria east and west, respectively, of the northern Mid-Atlantic Ridge.
\end{abstract}

\section{INTRODUCTION}

Information on the morphology of bacteriophages from marine habitats is as scant as that on marine phages in general. Smith \& Krueger (1954) were the first to describe a phage isolated from an estuarine environment. Further information concerning phages from estuarine waters or sediments were presented by Kakimoto \& Nagatomi (1972), Sklarow et al. (1973), Keynan et al. (1974), Zachary (1974), Hidaka \& Tokushige (1978), Nakamura et al. (1978), and Koga et al. (1982). A large portion of the phage strains described by the aforementioned authors was isolated with Vibrio parahaemolyticus as host which is not regarded as a marine bacterium.

Bacteriophages isolated from oceanic water or sediment were investigated mainly by Hidaka \& Fujimura (1971) and Hidaka (1973) who provided information on 32 out of 37 truly marine phage strains described so far. Other reports were presented by Valentine et al. (1966), Espejo \& Canelo (1968), Johnson (1968), Wiebe \& Liston (1968), and Stevenson \& Albright (1972).

All counted, some 60 bacteriophage strains from estuarine or oceanic habitats were morphologically characterized by the authors mentioned above. With one exception, these phages have a polyhedral head to which a tail is attached and, therefore, are ascribed to group A, B, or C of Bradley's (1967) classification. The exceptional phage 
PM2, isolated by Espejo \& Canelo (1968), has lipid-containing virions of hexagonal outline which do not reveal any tail structure (Silbert et al., 1969). Considerable variation was observed in the tailed phages concerning the length of head and tail as well as in regard to additional structures such as base plate, tail fibres, or pins attached to the tail tip.

Unspecified bacteriophages, derived by filtration of estuarine or oceanic water samples without subsequent propagation on host bacteria, were reported by Sieburth (1979) and Torrella \& Morita (1979). Most of the particles presented by these authors seem to belong to group A, a few to group B, and one to group C of Bradley.

Further information on the morphology of bacteriophages of marine origin will be presented in this paper. From about 300 phage strains, isolated from water samples collected in the North Sea near Helgoland and in the Atlantic at 42 stations between the European continental shelf and the Sargasso Sea (Moebus, 1980), 75 were selected for this investigation. The host ranges of these phages were reported by Moebus \& Nattkemper (1981) and Moebus (1983). All but a few strains are infective for members of the family Vibrionaceae (Moebus \& Nattkemper, 1983).

\section{MATERIAL AND METHODS}

Me dia : Seawater agar, soft seawater agar, seawater bouillon (SWB/5), and seawater mixture were of the same composition as given by Moebus \& Nattkemper (1981).

Bacteria and bacteriophages: The same designations are used as by Moebus \& Nattkemper (1981). Strains characterized by the capital letter $\mathrm{H}$ were derived from seawater sampled near Helgoland (depth $1 \mathrm{~m}$ ), and strains of the A-series were isolated from Atlantic Ocean water collected at the depth of $6 \mathrm{~m}$. With few exceptions, the phages were isolated from the same water sample as the respective host bacteria.

For the purpose of this investigation, phage strains were selected which yield high titre phage stocks when standard methods are employed. The investigated A-series phages were recovered from the various regions sampled in the Atlantic (Moebus, 1980). Their hosts belong to 10 groups of bacteria, 9 of which combined between 2 and 128 isolates the generic relationship of which was revealed by phage-host cross-reaction tests (Moebus \& Nattkemper, 1981; Moebus, 1983). The tenth group comprised bacteria which were lysed by one phage strain only.

Preparation of high-titre phage suspensions for electron microscopy: Starting with single plaques, fresh phage stocks were prepared by a standard plate-elution method (Moebus, 1980). These were then used to inoculate $50 \mathrm{ml}$ batches of SWB/5 containing about $10^{8}$ cells $\mathrm{ml}^{-1}$ of the respective host bacteria. The multiplicity of infection was about 1 . These cultures were incubated in a shaking water bath. Plates and liquid cultures were incubated at $25^{\circ} \mathrm{C}$.

After lysis the cultures were centrifuged for $30 \mathrm{~min}$ at about $4500 \times \mathrm{g}$ and $10^{\circ} \mathrm{C}$ and the recovered supernatants stored in the refrigerator overnight. To concentrate the phage particles, the supernatants were centrifuged for $2 \mathrm{~h}$ at $39000 \times \mathrm{g}$ and $4^{\circ} \mathrm{C}$ and the pellets suspended in $2 \mathrm{ml}$ of sterile seawater mixture. Finally, these suspensions were centrifuged at low speed as stated above and the supernatants transferred into sterile screwcap tubes and stored in the refrigerator.

Preparation for electron microscopy: Carbon coated Pioloform F films 
mounted on 400 mesh copper grids were used throughout. Immediately before use, the grids were glow-discharged to obtain a hydrophilic surface of the supporting film. A drop of phage suspension was placed on the grid and sucked off with filter paper after 1 min. Then the grid was washed three times with distilled water. Finally, the specimen were negatively stained, generally by applying a drop of $1 \%$ uranyl acetate $(\mathrm{pH} 4.3)$ which was removed after 30 sec with filter paper. With several phage strains negative staining with phosphotungstic acid (1\%, pH 7.2) was additionally employed. Electron micrographs were taken with a Siemens Elmiscope IA operating at $80 \mathrm{kV}$ with a nominal magnification of 40000 . The real magnification (about $10 \%$ higher) was determined as described by Frank \& Day (1970).

Determination of type of bacteriophage nucleic acid: Bacteriophage suspensions with a titre of at least $10^{11}$ plaque forming units $\mathrm{ml}^{-1}$ were treated according to the method of Bradley (1966). However, with strain H17/1 the treatment of phage suspension with DNase and RNase before fixation was at $15^{\circ} \mathrm{C}$ for $4 \mathrm{~h}$ and $60 \mu \mathrm{g} \mathrm{ml}^{-1}$ of both enzymes.

\section{RESULTS}

The bacteriophage strains investigated are grouped according to morphological similarities (Table 1). Apart from the assignment to one of the Bradley groups A, B, or C, the various groups differ by the lack or existence of appendages such as antennae or (long) tail fibres tipped with conspicuous structures, which are less commonly encountered than a base plate with (short) fibres or spikes attached.

Some appendages are fragile structures which may be lost, at least partially, during the preparation of phage suspensions for electron microscopy. Therefore, the possibility exists that strains described as lacking such appendages do have them in reality. There are indications that the appendages of some strains were easily lost while with others seemingly no loss occurred of the same type of structure. Also, with a few strains many more burst heads were observed than undamaged ones, while the heads of most phage strains remained more or less intact even if flattened.

Due to the large number of phage strains investigated, it was impossible to test other methods than those described to elucidate the presently somewhat unsatisfactory observations made with a number of strains. Specific information on these points will be presented in the description of the various morphological groups which follows the order presented in Table 1. In Table 2, the measurements of the phages' components are compiled, and information on the type of nucleic acid is also presented if available.

Regarding the phage dimensions it must be pointed out that measurements taken from negatively stained particles are prone to error. This concerns especially phage heads which, owing to the influence of surface tension, may be flattened during the drying process, thus appearing larger than they really are. The degree of this type of distortion may vary between different phage strains. With some strains belonging to Bradley's group A, only one to three undamaged particles could be measured, because in these cases the vast majority of particles was found with contracted sheath or broken-off tail. In any case, only particles that appeared perfectly stained and undistorted were taken into account. 
Table 1. Grouping of the investigated bacteriophage strains according to morphological characteristics, with the figures presenting typical phage particles of the respective group indicated

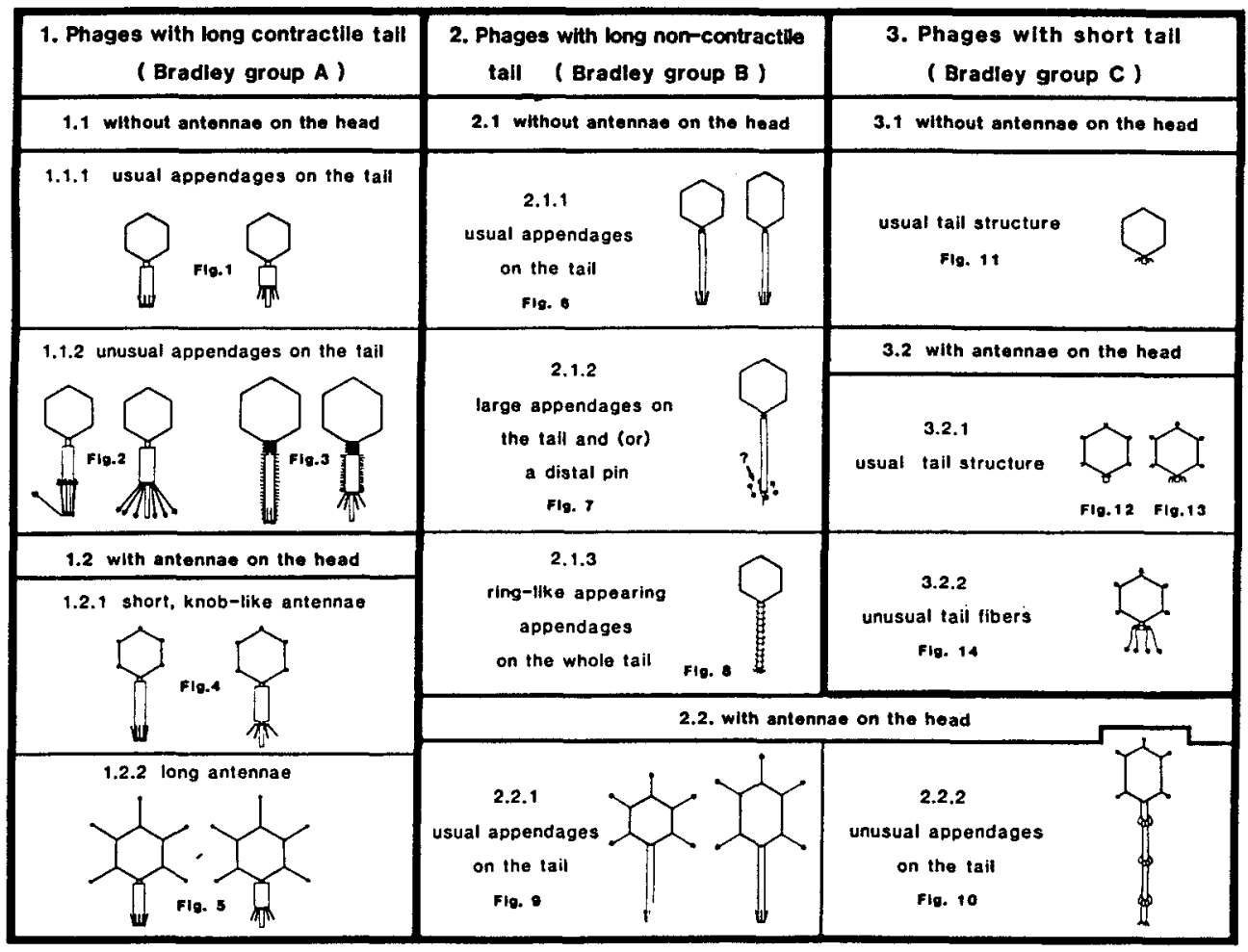

Phages with long contractile tail (Bradley group A)

In phage strains belonging to this group, only virions with isometric head were observed.

\section{Phages with no antennae on the head}

Phages with usual appendages on the tail

This group comprises 24 of the phage strains investigated. It is by far the largest of the 12 groups. The tail of the phages is about as long as or slightly longer than the head. In intact particles, the existence of a base plate was indicated in only a few cases (Fig. 1d); however,'this structure was visible in virions of almost all strains with a contracted sheath (Fig. 1f, compare with e). In this case, the base plate usually appears to be separated from the sheath by a small space.

All strains of this group have short appendages attached to the tip of the tail. In five strains (A139/3, A159/1, A660/1, A852/1, and A1152/1), these structures always point downward (Fig. 1d and e), resembling spikes such as those known for $T$-even phages. In the remaining strains, these appendages with intact particles were found to point upward in a fashion discernible with the virion shown in Figure 1b. After contraction of the sheath these structures point downward, indicating that they are short fibres (Fig. 1c). 


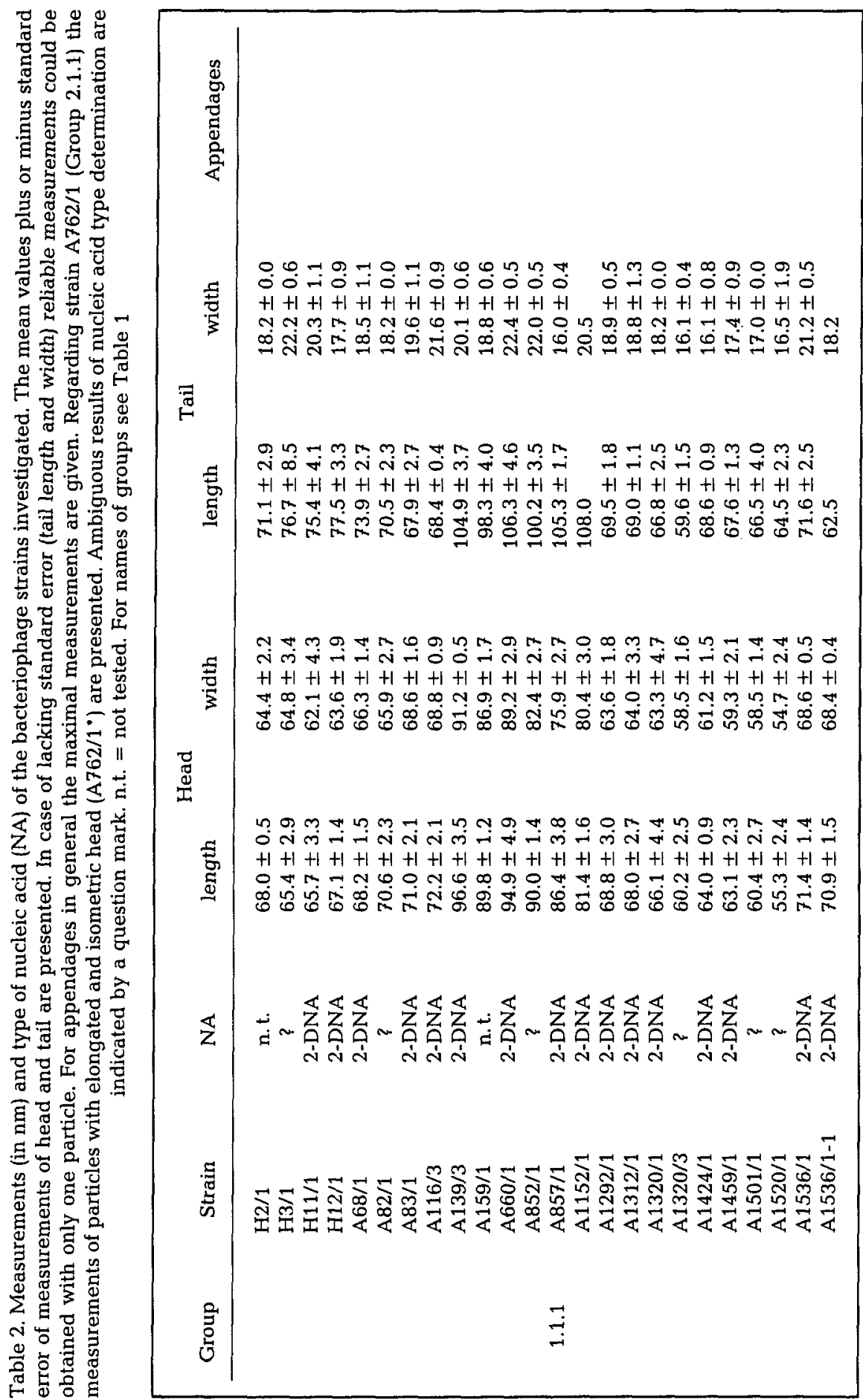




\begin{tabular}{|c|c|c|c|c|c|c|}
\hline 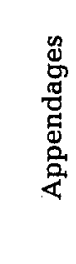 & 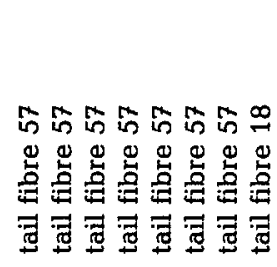 & 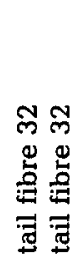 & & 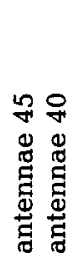 & & 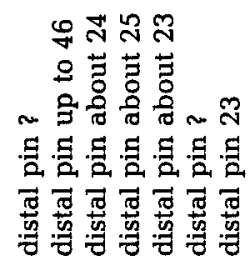 \\
\hline 胥 & 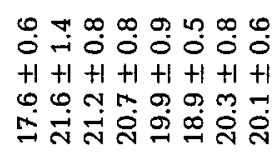 & 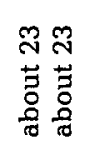 & 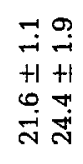 & 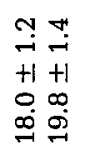 & 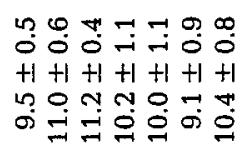 & 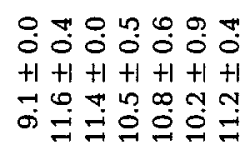 \\
\hline $\begin{array}{l}\text { 莺 } \\
\text { Ф }\end{array}$ & 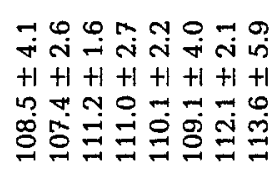 & 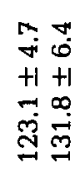 & $\begin{array}{l}m \\
+ \\
+1 \\
\dot{m} \\
\rightarrow \\
\rightarrow\end{array}$ & 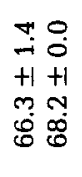 & 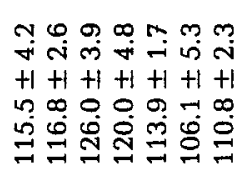 & 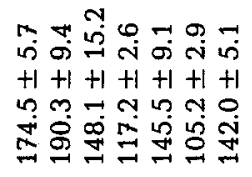 \\
\hline$\frac{\vec{z}}{\overline{7}}$ & 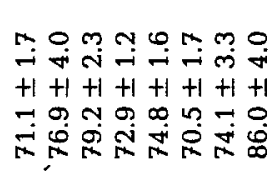 & 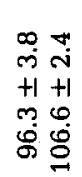 & 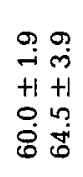 & 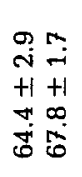 & 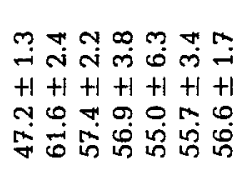 & 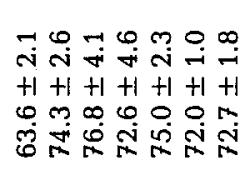 \\
\hline 莺 & 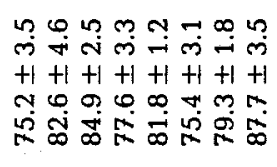 & 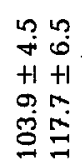 & 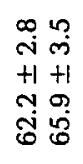 & 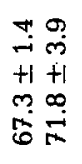 & 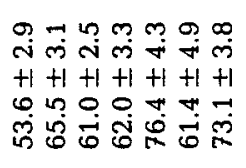 & 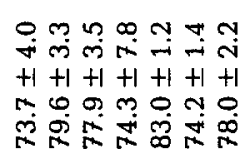 \\
\hline$\overleftrightarrow{z}$ & 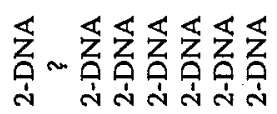 & 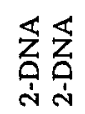 & 荾 & $\sim \overbrace{\text { ลุ่ }}^{\overleftarrow{\Delta}}$ & 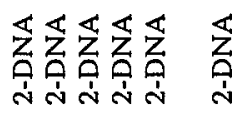 & 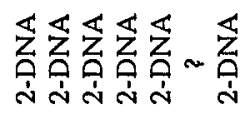 \\
\hline 吉 & 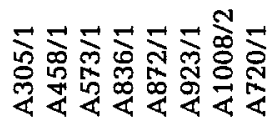 & 空点 & 蒠 & 突志 & 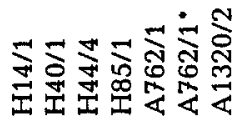 & 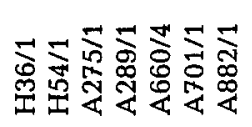 \\
\hline 을 & $\underset{-}{\stackrel{\sim}{-}}$ & & $\stackrel{\vec{\sim}}{\rightarrow}$ & $\underset{\sim}{\underset{-}{N}}$ & $\overrightarrow{\vec{i}}$ & $\underset{\sim}{\stackrel{\sim}{\sim}}$ \\
\hline
\end{tabular}




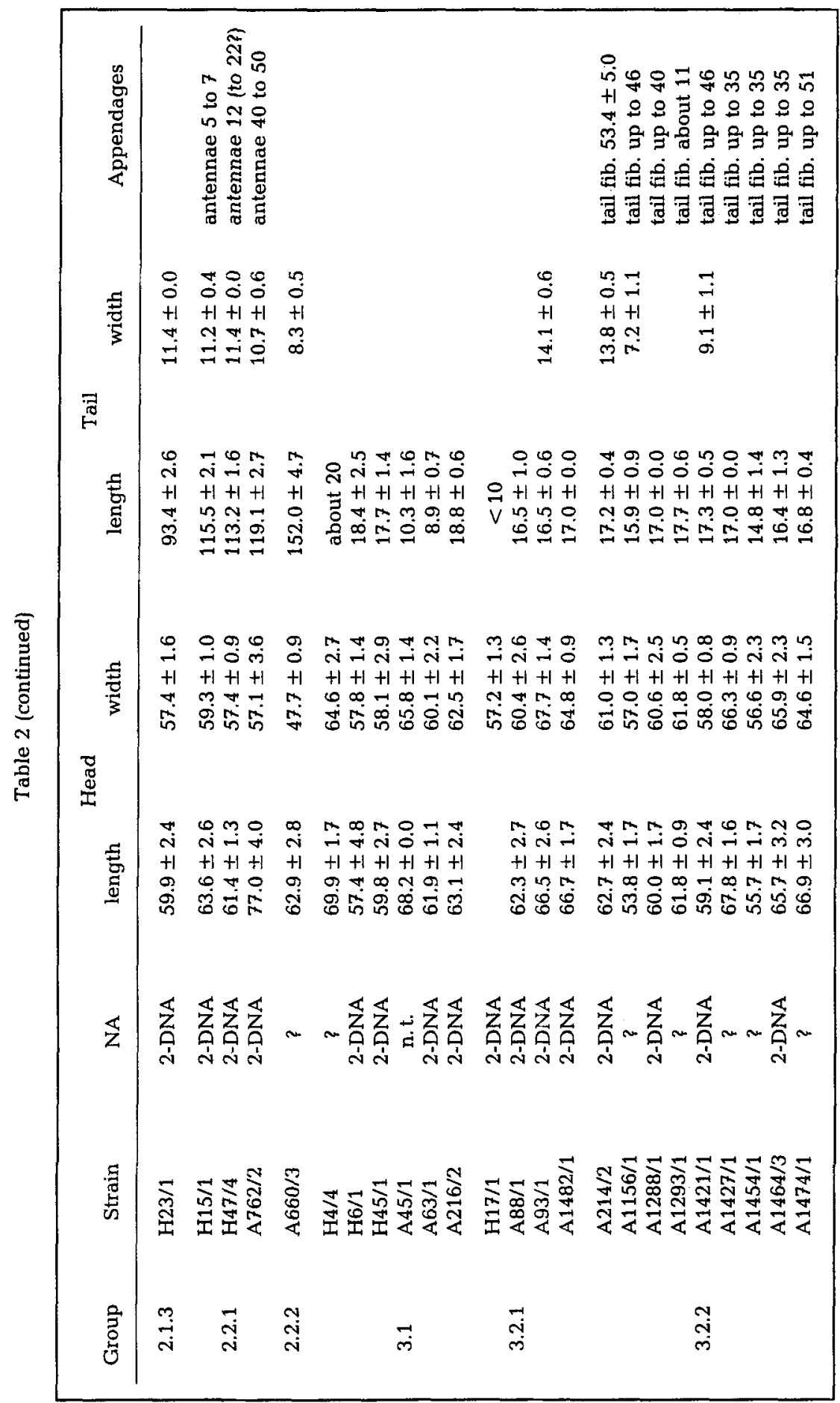



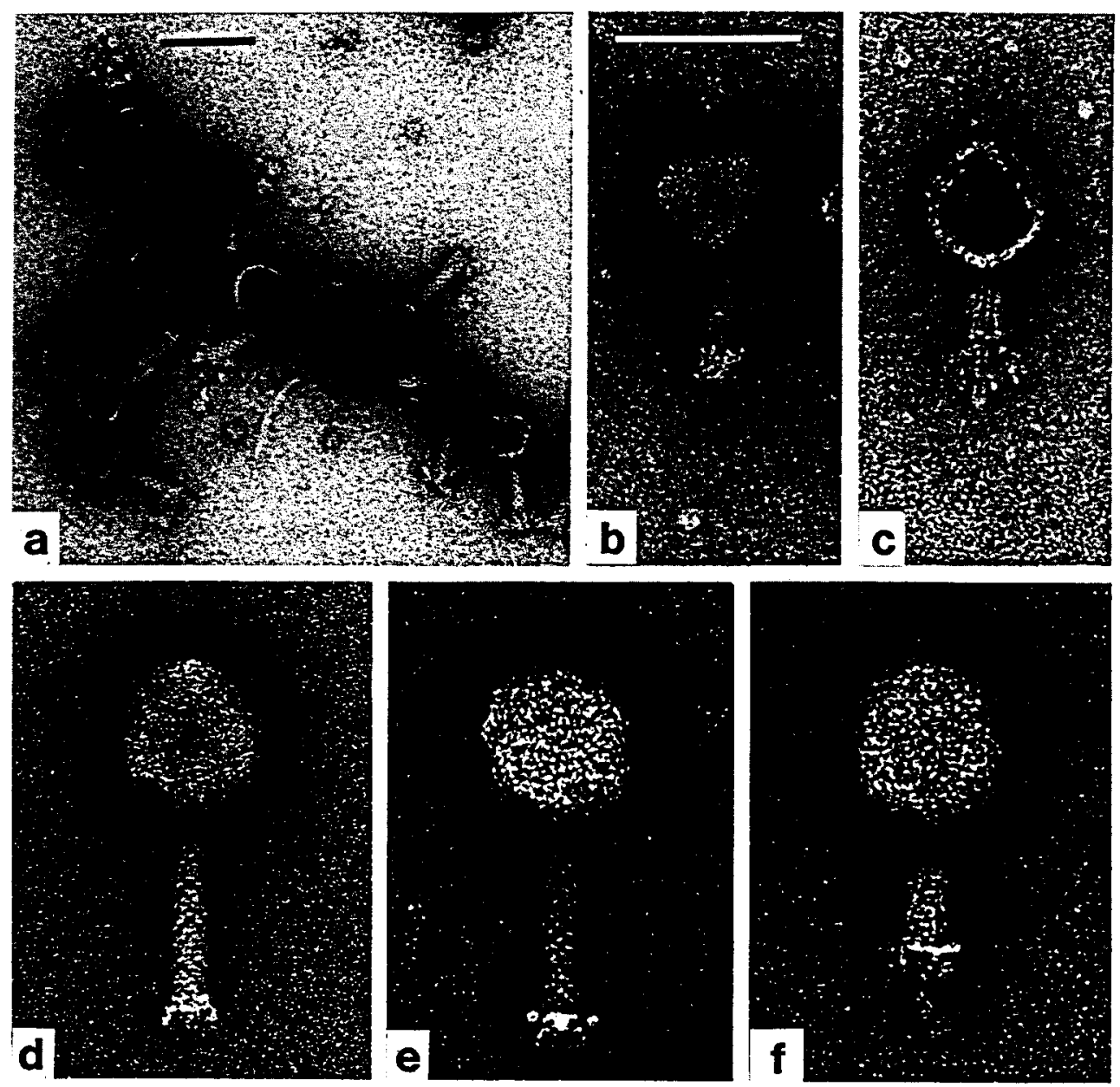

Fig. 1. Phages with usual tail appendages, negatively stained with uranyl acetate (UA). a: Phage H11/1. $b$, c: phage A1292/1. d: phage A852/1. e, f: phage A139/3. Bar represents $100 \mathrm{~nm}$

Phages with unusual appendages on the tail

This group combines phages with thread-like appendages attached to the tip of the tail which may bear globular bodies at the distal end. This common feature, however, comes in rather distinct configurations.

One sub-group comprises 8 strains of A-series phages such as shown in Figure 2 , with 7 of them virtually inseparable in terms of morphology: A305/1 (Fig. 2c and d), A458/1, A573/1 (Fig. 2b), A836/1, A872/1, A923/1 (Fig. 2a), and A1008/2 (Fig. 2e). Their

Fig. 2. Phages with unusual tail appendages, negatively stained with UA. a: phage A923/1. $b$ : phage A573/1. $c$, d: phage A305/1, with arrows in $d$ pointing to structures at the end of fibres which upon detachment from tail apparently change configuration. e: phage A1008/2. $-h$ : phage A720/1, with arrows in $f$ pointing to antennae-like structures assumed to be artifacts. Bar represents $100 \mathrm{~nm}$ 

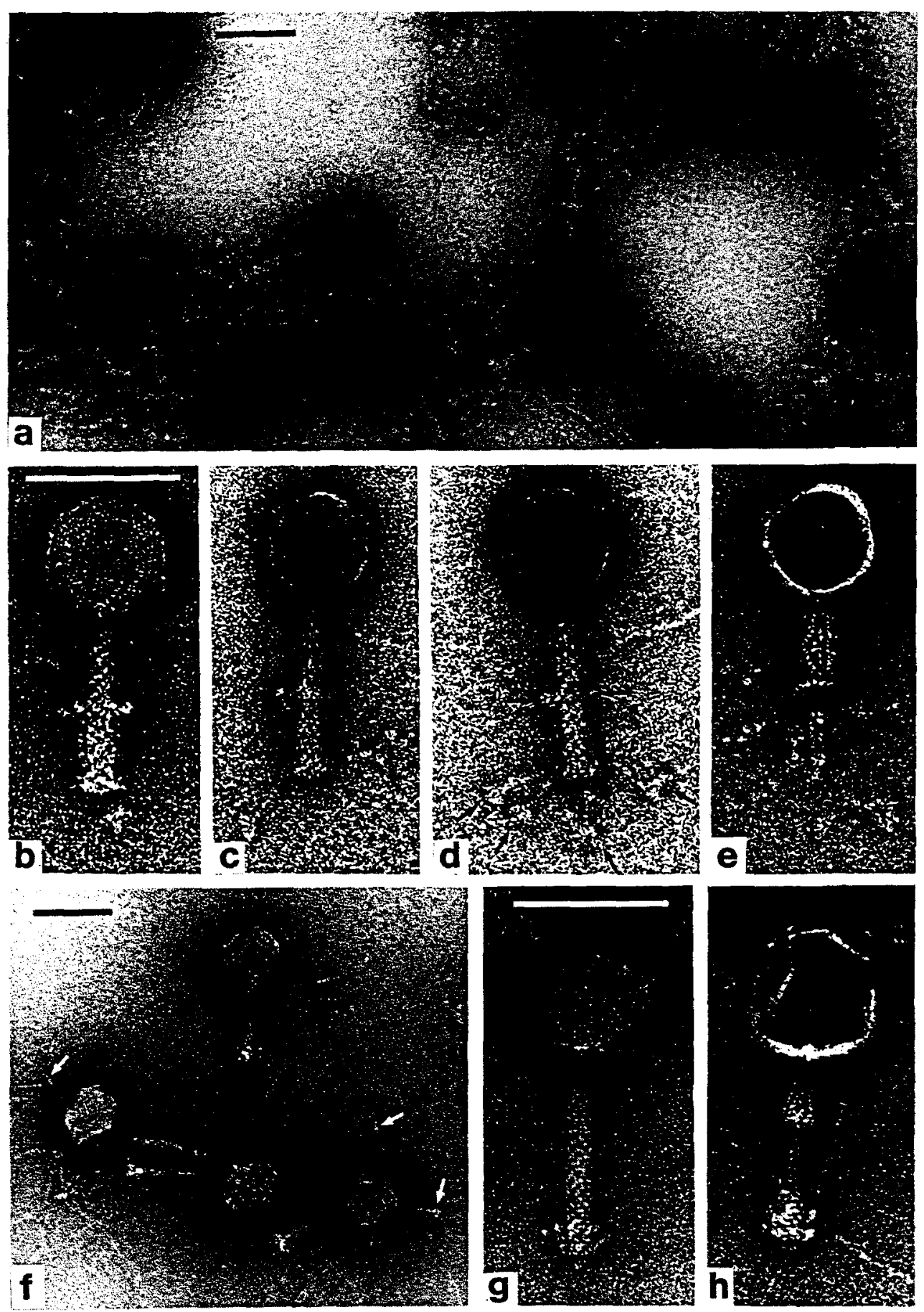
appendages, extending about half the tail length toward the head, are thin threads (Fig. 2a-d) tipped with round structures which, after detachment from the tail, unfold to a lobate (trilobate?) configuration (Fig. 2c and d). The virions most probably have 6 of these appendages each (Fig. 2d), and it seems likely that the fibres are attached to a base plate (Fig. 2e). A similar arrangement of tail fibres has been reported by Bradley (1967) for the Pseudomonas phage PB-1; however, knob-like structures at the end of its fibres are missing.

A base plate was discernible only after contraction of the sheath, which also holds true for the eighth strain, A720/1. It differs from the aforementioned ones mainly in the shortness of its tail fibres (Fig. $2 \mathrm{f}-\mathrm{h}$ ). The filamentous material seen in Figures $2 \mathrm{f}$ and $2 \mathrm{~h}$ in the vicinity of virions was commonly found in preparations of this phage strain. Its origin and nature remains obscure. However, from all observations made we must conclude that strain A720/1 does not have antennae, as might be assumed for the two particles shown in Figure $2 \mathrm{f}$ (below left and right).

The second sub-group (Fig. 3) comprises only two strains, H7/2 and H84/1, which are almost indistinguishable morphologically. There are indications that the comparatively thick and rigid fibres, attached to the tail end (Fig. $3 \mathrm{~g}$ ), in fully intact particles are aligned with the tail (Fig. 3c) as found in the fibres of the phages of the first sub-group (Fig. 2). The existence of a base plate seems to be indicated (Fig. 3d and $\mathrm{k}$ ) but remains doubtful. This also relates to the original arrangement of the globular structures seen in the microphotographs. The particle presented in Figure 3c may show such structures in their original position, but clearly provides no sufficient evidence.

Probably over the full length of the contractile sheath the tail is surrounded by short fibrous material (fringes) which, upon contraction of the sheath, becomes more conspicuous (Fig. $3 \mathrm{~d}$ and i). Due to these fringes, it is impossible to determine the actual width of the tail. Configurations such as shown in Figures $3 e$ and $3 f$ may be disks of disintegrated sheaths. Where tails are broken-off, the adaptor protein securing the linkage between tail and head, which differ in symmetry, is apparent (Fig. 3h and $\mathbf{k}$, arrowheads). Adjacent to this a collar consisting of three disks is seen, as indicated by arrows (Fig. $3 b-d$ and $h$ ).

The origin and nature of the fuzzy material surrounding the head of almost all particles scrutinized remain obscure. It may represent antennae which in this case would appear to be branched (Fig. 3b). However, there is no unquestionable evidence to support this interpretation.

Fig. 3. Phages with unusual tail appendages, negatively stained with UA. a-d: phage H7/2. Small arrows in $b-d$ indicate the 3 disks of the collar, large arrow in $c$ points to a configuration resembling those found with phages shown in Fig. $2(b-d)$, arrowhead in $d$ indicates adaptor protein linking head and tail. e, $f$ : disks of contracted sheath protein of phage $H 7 / 2$ (e) and phage H84/1 (f), revealing fringes of tail. $g$ : tail of phage $\mathrm{H} 84 / 1$ with 6 thick fibres attached to tail end, arrow indicates a fibres masked for the greater part. $h$ : tail of phage $\mathrm{H7} / 2$ with adaptor protein (arrowhead) and collar (arrows). $i, k$ : phage $\mathrm{H} 7 / 2$, tails with contracted sheath, with arrowhead and arrow in $k$ marking adaptor protein and a structure possibly representing a base plate, respectively. Bar represents $100 \mathrm{~nm}$ 

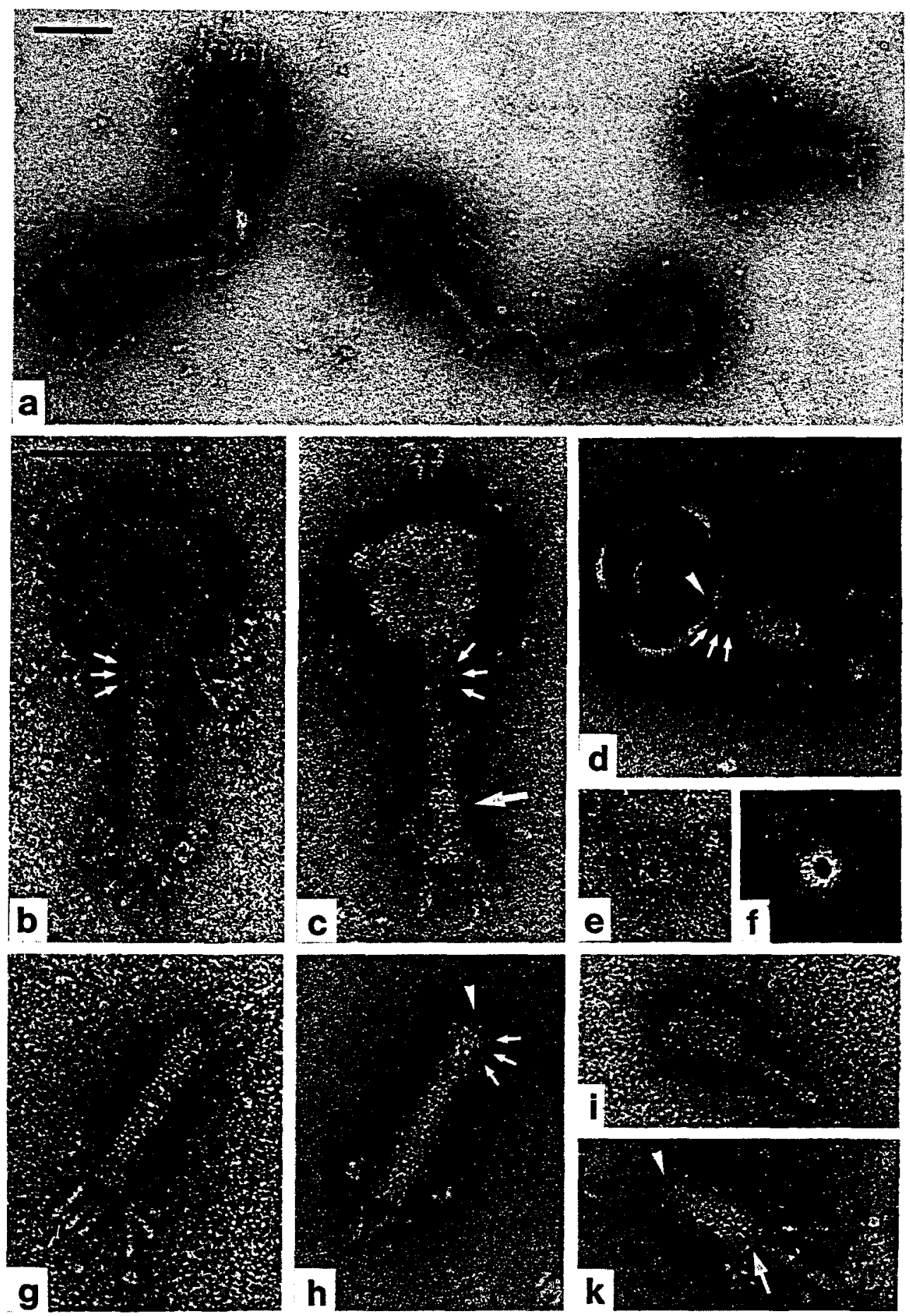


\section{Phages with antennae on the head}

\section{Phages with short knob-like antennae}

In addition to the knob-like antennae of unknown structure which appear to be directly attached to the vertices of the head (Fig. $4 \mathrm{~b}-\mathrm{d}$ ), the two strains assigned to this group differ from the strains described on page 388 with regard to their longer tails but resemble those phages in other aspects.

In phage A1350/1, of more than 100 particles only a few with uncontracted sheath were found (Fig. $4 a$ and b). Tail length is about 1.5 times the length of the head (Table 2). A base plate was never discernible; however, short spike-like structures on the tail's tip
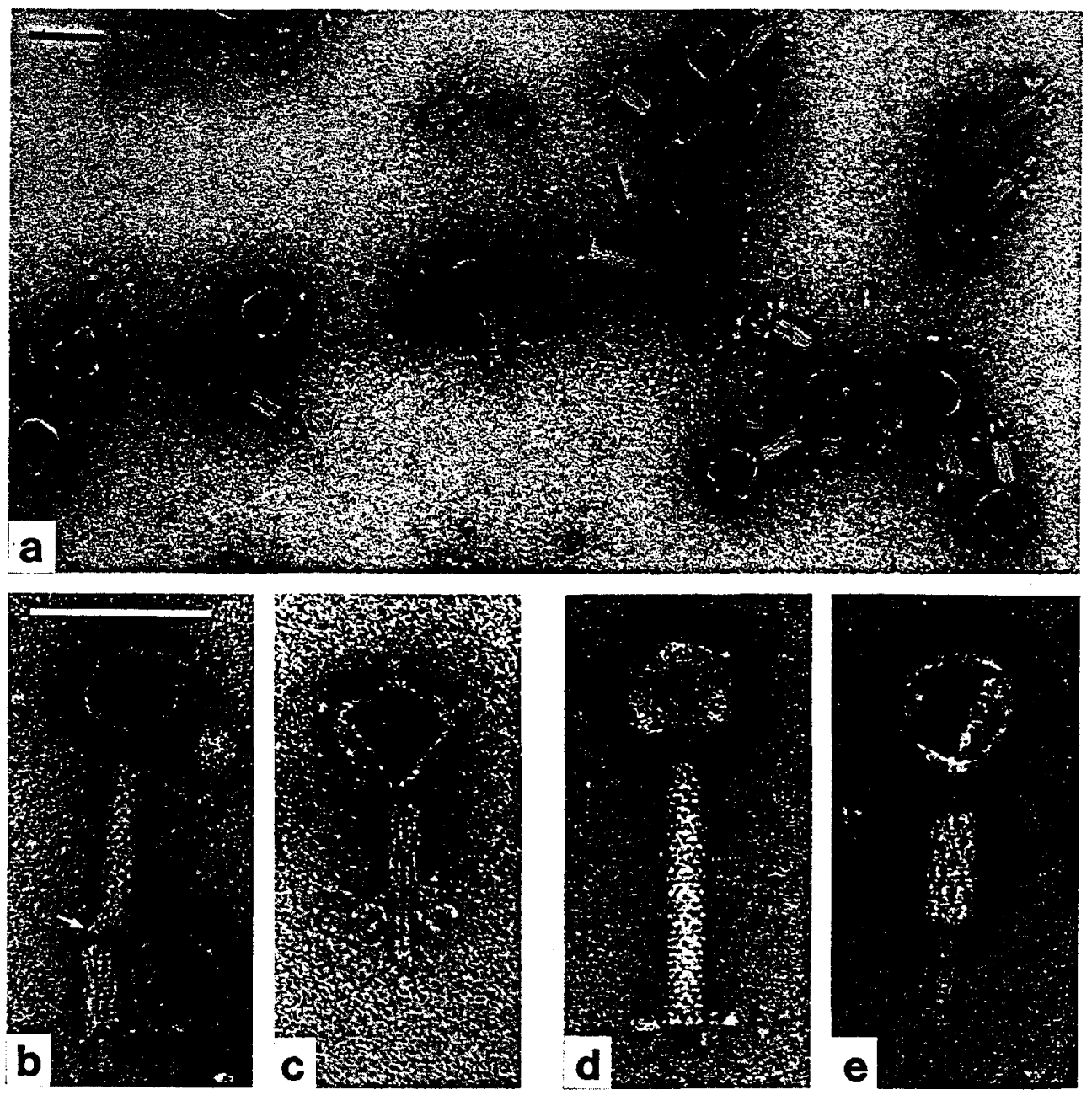

Fig. 4. Phages with short knob-like antennae, negatively stained with UA. a-c: phage A1350/1, arrow in a pointing to one of the rarely observed particies with native tail structure. Arrow in $b$ points to tail end of particle. d, e: phage H96/1. Bar represents $100 \mathrm{~nm}$ 
can be seen. Particles with contracted tail give the impression of a sheath unravelling upon contraction (Fig. $4 \mathrm{a}$ and $\mathrm{c}$ ).

In contrast, in intact particles of the other strain, H96/1, the existence of a base plate with spike-like appendages is indicated. Its tail is about 2.5 times as long as its head. The antennae of strain H96/1 are very small but were seen in the majority of the particles.

\section{Phages with long antennae}

The only essential difference between the two members of this group and the phages presented on p. 388 is the existence of long antennae extending from the vertices of the head. The antennae are tipped with trilobate bodies (Fig. $5 \mathrm{~b}-\mathrm{d}$ ). Short appendages attached to the tail end usually point upward or slightly sideward, resembling those observed in the majority of phages with the usual tail appendages (Fig. 5a and b,
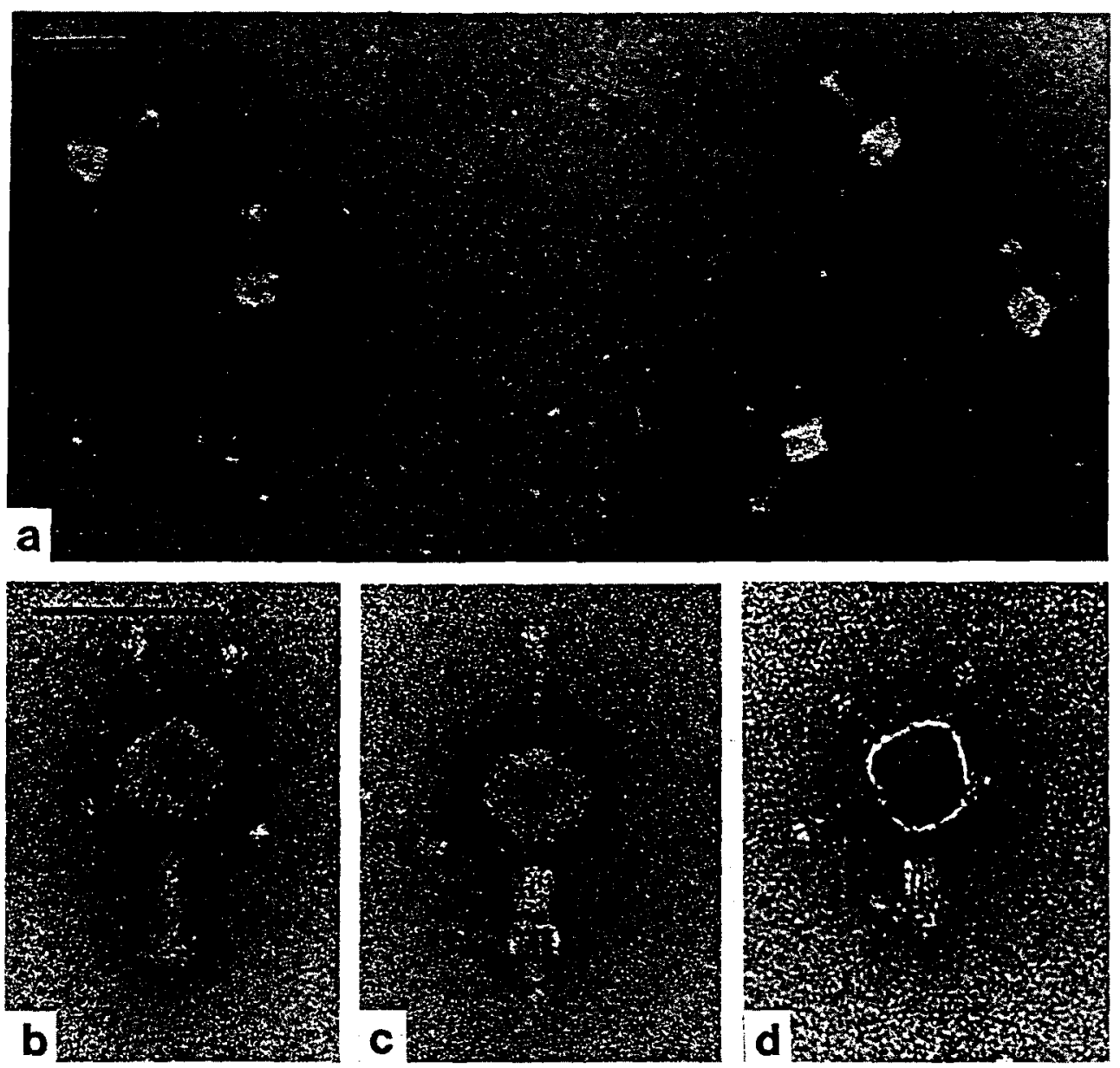

Fig. 5. Phages with long antennae, negatively stained with UA. a-c: phage A1494/1. d: phage A1423/2. Bar represents $100 \mathrm{~nm}$ 
compare Fig. 1b). A structure probably representing the base plate was visible in particles with contracted sheath (Fig. 5c).

\section{Phages with long non-contractile tail (Bradley group B)}

Among the phages belonging to this group a few strains were found, the particles of which have an elongated head. In two strains, virions both with elongated or isometric head were observed. Isometric heads, however, are characteristic for the majority of the phage strains assigned to this group.

\section{Phages with no antennae on the head}

Phages with usual appendages on the tail

Six strains are combined in this group. In strain A1320/2 (Fig. 6e) only elongated heads were observed, while in strain A762/1 (Fig. $6 a, c, d$ ), up to $10 \%$ isometric heads were found besides elongated ones. The remaining four strains (all of the $\mathrm{H}$-series, see Table 2) are characterized by isometric heads.

A common feature of the six strains is a slender flexible tail with short rigid fibres attached to its tip. In strain H14/1 (Fig. 6b), the original arrangement of these fibres remains obscure; the irregularity observed in their position is regarded as an artifact. In the other strains, numerous particles were observed with the fibres regularly arranged, forming a structure which appears in either a rectangular (Fig. 6c, d) or a triangular configuration (Fig. 6e, g). Mostly both of them are present in virions of the same strain. Corresponding observations were made by Valentine et al. (1966) in a phage strain isolated by Spencer (1960) from the North Sea, which also belongs to the Bradley group B. Occasionally, a collar was discernible, formed by one disk just beneath the head (Fig. $6 \mathrm{~d}, \mathrm{e}$, arrow), and sometimes the structure involved in the linkage between head and tail was visible in empty heads (Fig. $6 \mathrm{~g}, \mathrm{~h}$, arrowhead).

Phages with large appendages and (or only) a distal pin on their tail

The seven bacteriophage strains included in this group are characterized by an isometric head and a long flexible tail which extends into a single rigid pin. In this regard, these phages resemble the coliphage T5. There may or may not be a number of globular bodies either directly attached to the tail or at the tip of short fibres extending from the end of the tail.

The most complex composition of virions was observed in strains A275/1, A660/4, and $A 882 / 1$, which appear to be morphologically identical. Apparently, six short fibres tipped with lobate structures (Fig. $7 \mathrm{~b}, \mathrm{~g}$ ) extend from the tail at the same region as the central pin. Usually, the latter could be seen only together with particles, the appendages of which were either relocated or (partially) lost during preparation (Fig. $7 a$, arrows, and $7 \mathrm{c})$.

The particle shown in Figure $7 \mathrm{c}$ is possibly an example of relocation of appendages in toto. If so, such a relocation can occur only if the globular bodies are bound together in some way, forming a 'bracelet' configuration that keeps them in position during the process. The configurations shown in Fig. $7 \mathrm{~g}$ do not contradict such an assumption. 

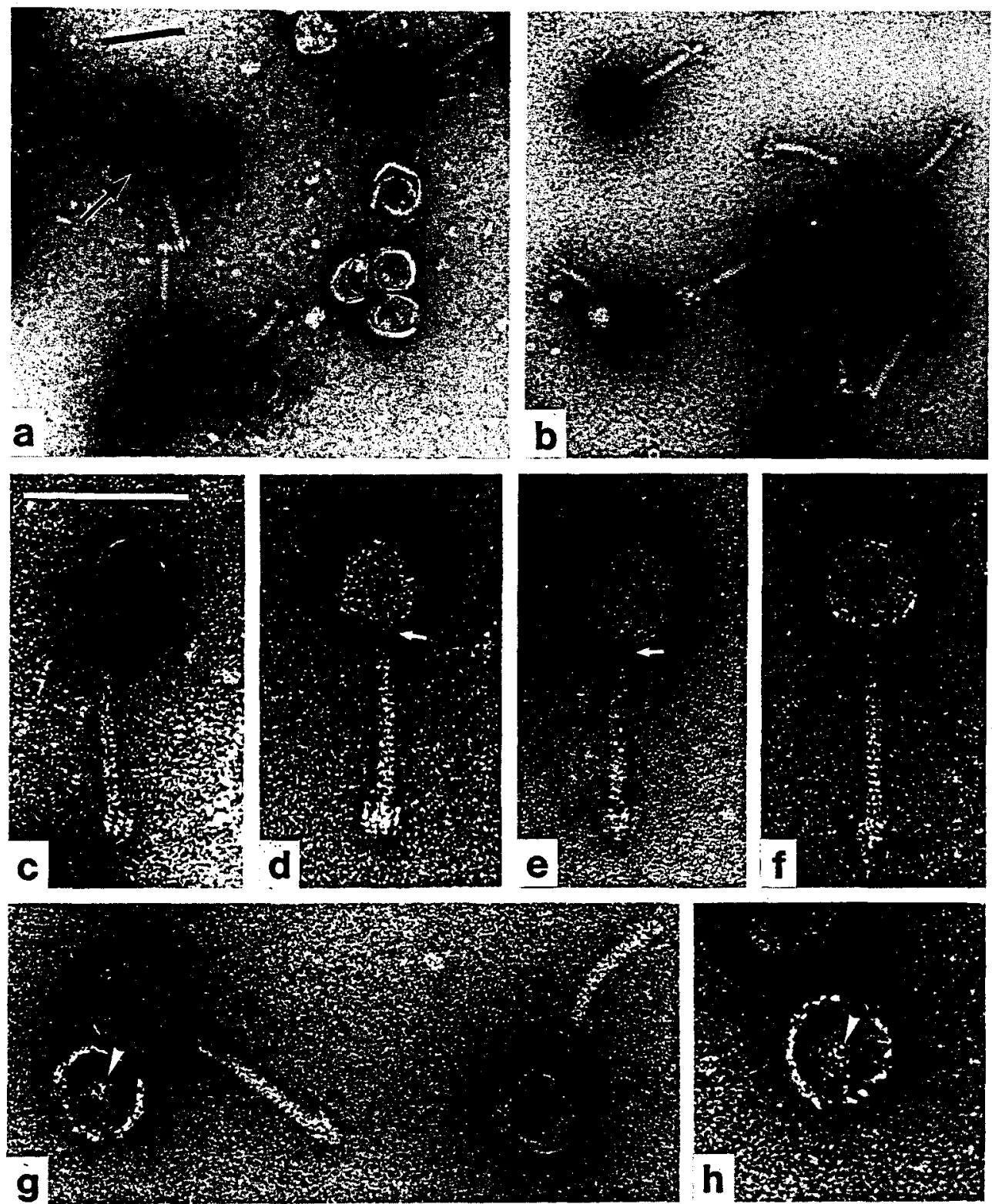

Fig. 6. Phages with usual tail appendages, negatively stained with UA. $a, c$, and $d$ : phage A762/1 arrow in $a$ indicates particle with isometric head. $b$ : phage H14/1. e: phage A1320/2. Arrows in $d$ and $e$ mark faintly visible collar with one disk. $f$ and $h$ : phage H40/1. g: phage H85/1. Arrowheads in $g$ and $h$ indicate point of linkage between head and tail, in topview of empty heads. Bar represents $100 \mathrm{~nm}$ 

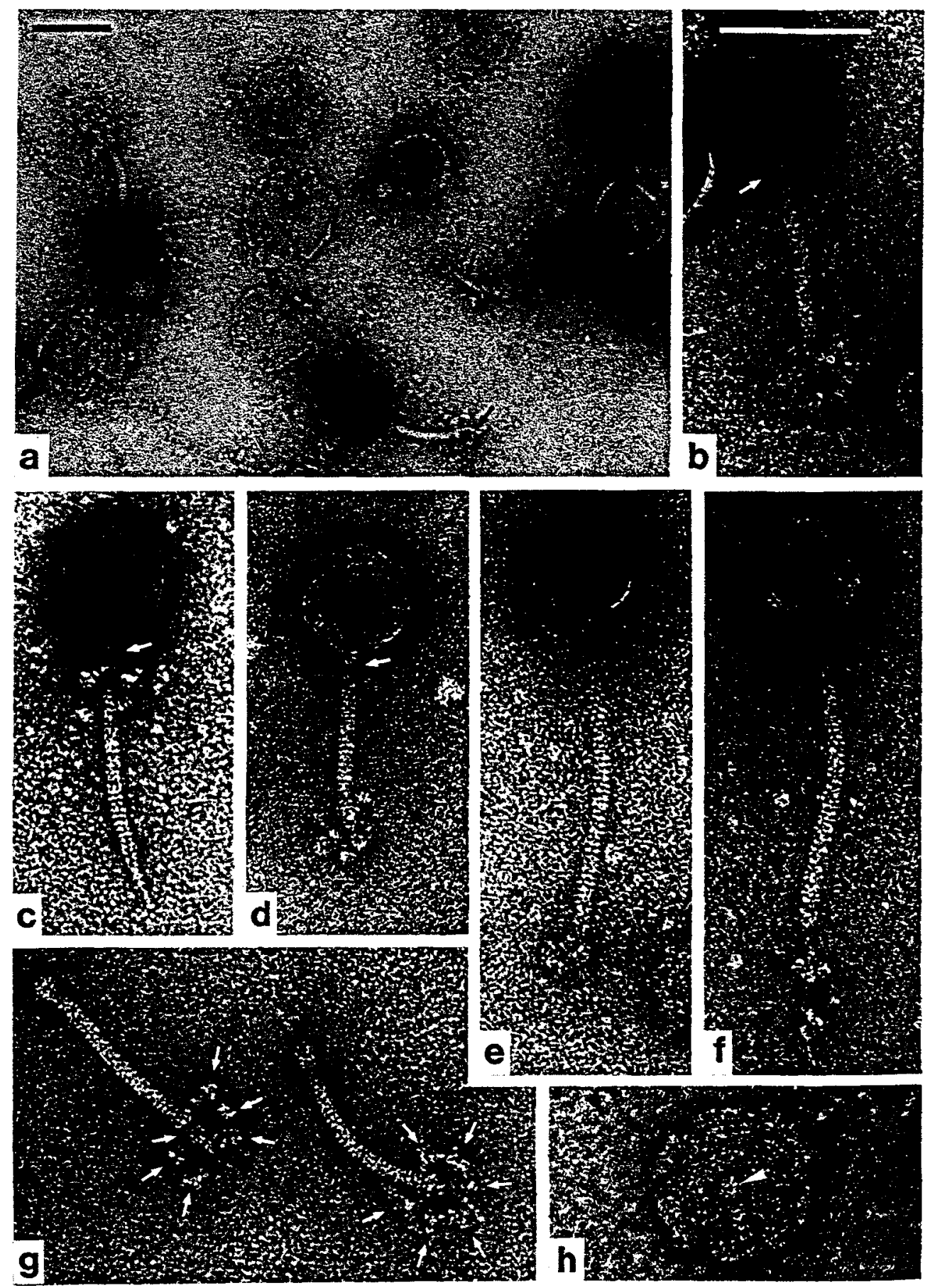
Strain A701/1 (Fig. 7d) closely resembles the aforementioned ones, its slightly shorter tail apparently being the only morphological difference.

Phage H36/1 (Fig. 7e and f) differs from the four A-series phages mentioned above by its considerably longer tail. The pin seems to break off easily. Likewise, the attachment of the globular bodies to the tail's tip was obviously less stable than observed in the aforementioned A-series strains.

In phage strains A289/1 and H54/1, both exhibiting the distal pin, no globular or lobate appendages of the tail were observed. However, the existence of such appendages cannot be completely ruled out. In micrographs of these phages numerous structures are visible which in size and form correspond with the appendages of the phages described above.

A high incidence of burst heads was observed in the bacteriophages of this group, except in strain A289/1 (Fig. 7a). This is a unique feature in the 12 groups of phage strains investigated. Burst heads often reveal the structure of the point of attachment of the tail (Fig. $7 \mathrm{a}$ and $\mathrm{h}$, arrowheads).

Phages with rings of small appendages along the whole length of the tail

Strain $\mathrm{H} 23 / 1$ has an isometric head with a non-contractile tail of low flexibility. Along its whole length, the tail bears regularly spaced ring-like assemblages of small structures which appear to have the shape of thorns (Fig. 8d), their tip directed at an angle of about $45^{\circ}$ toward the head. The number of these assemblages is most probably eleven. They seem to be easily lost (Fig. $8 \mathrm{~d}-\mathrm{f}$ ) and the virion as a whole appears to be rather sensitive to the preparative methods employed (Fig. 8a, b). The structure of the tail end remains dubious. Although the tail's tip, with many particles, looks different from the upper part of the tail, neither a base plate nor spikes or short fibres were clearly discernible (Fig. 8d-f).

Phages with antennae on the head

Phages with usual appendages on the tail

Except for the presence of antennae, the three bacteriophage strains of this group do not significantly differ from those described on p. 398. As in strain A762/1 (see above), the majority of the virions of strains $A 762 / 2$ have an elongated head; the portion of virions with isometric head, however, was smaller in the latter strain. Irrespective of the head's shape, long antennae tipped with trilobate structures were present, most probably extending from the vertices (Fig. 9a, c, and d). Strain H47/4 (Fig. 9b, e, and f) has an isometric head with shorter antennae. These bear conspicuous bodies on the tip, the structure of which, however, remains unknown. Strain H15/1 has an isometric head with antennae which apparently are either short stems or knob-like structures. Regarding the tail-end configuration of these strains, essentially the same observations were made as presented above.

Fig. 7. Phages with large tail appendages and (or only) a distal pin, negatively stained with UA. a: phage A882/1, arrowheads marking point of linkage between head and tail (see also Fig. $h$ ). $b, c$ : phage A275/1, particle shown in $c$ assumed to be an artifact (see text). d: phage A701/1. Arrows in $b, c$, and $d$ indicate faintly visible collar. e, $f$ : phage H36/1. $g$ : tails of phage A882/1, arrows indicate trilobate (?) end structure. $h$ : empty head of phage A660/4, arrowhead indicates point of linkage between head and tail (adaptor protein?). Bar represents $100 \mathrm{~nm}$ 

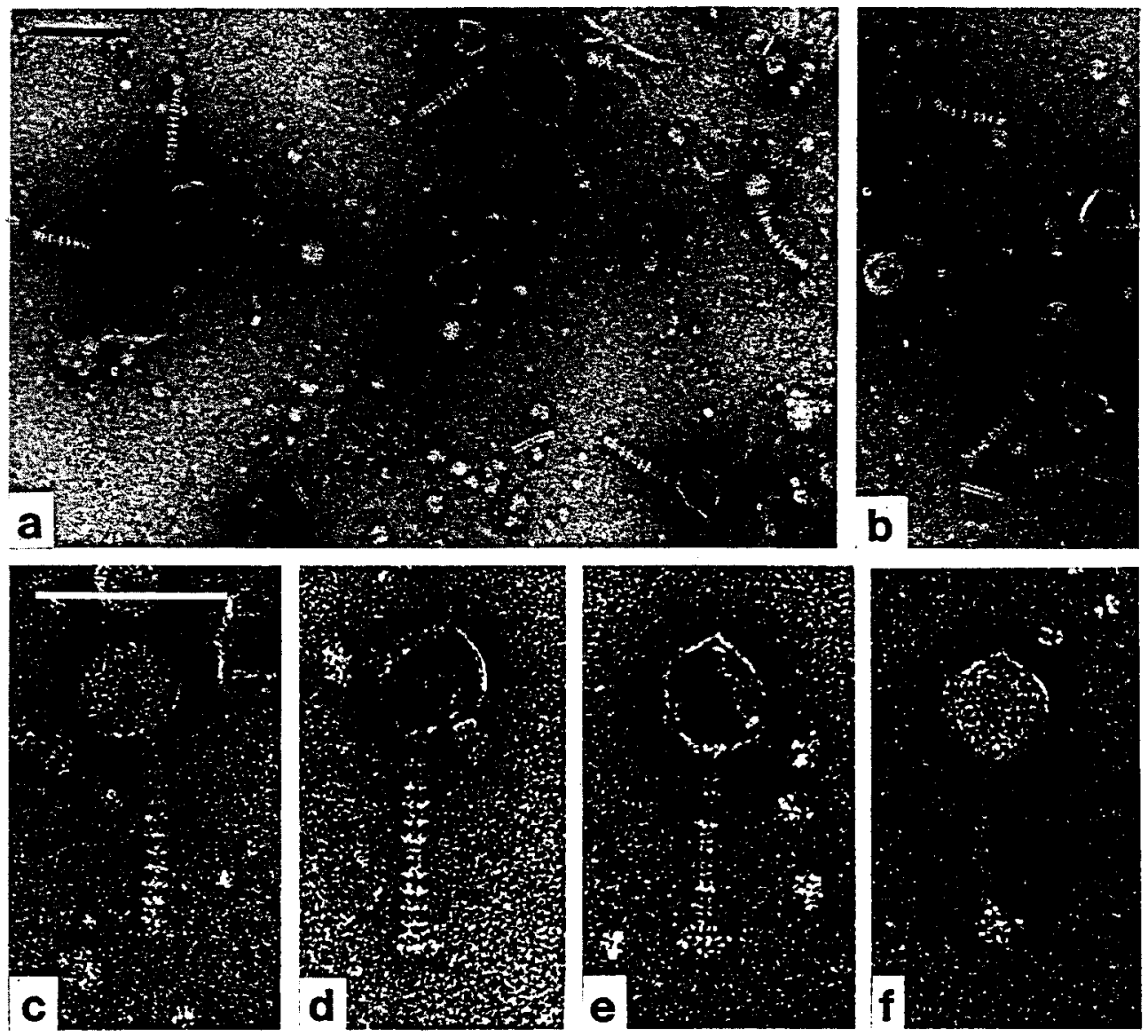

Fig. 8. Phage $H 23 / 1$, the only phage with rings of small appendages along the whole length of the tail, negatively stained with UA. The tail bears 11 regularly spaced ring-like structures, seemingly built of easily lost subunits resembling thorns pointing upward. Bar represents $100 \mathrm{~nm}$

Phages with unusual appendages on the tail

The only member of this group, phage strain A660/3, has an elongated head of hexagonal outline with short antennae on the vertices (Fig. 10a, b, arrows). The structure of the antennae remains unknown. Attached to the flexible tail, possibly by short fibres, there are three regularly spaced clusters of spherical or (more probably) lobate bodies (Fig. 10e-g). The tail tip of intact virions (Fig. 10c) appeared swollen with no appendages of any kind discernible. As in strain H23/1 (p. 401), the tail of phage A660/3 was easily penetrated by stain, preferentially if phosphotungstic acid was used, thus appearing as an empty tube. The adaptor protein (Fig. 10f, arrowhead) was visible in situ with numerous empty heads (Fig. 10d, arrowhead; see also 10a, b).

Ritchie et al. (1970) reported a phage derived from rumen fluid that resembles strain A660/3 in having 4 (or 5?) regularly spaced clusters of small appendages attached to a 

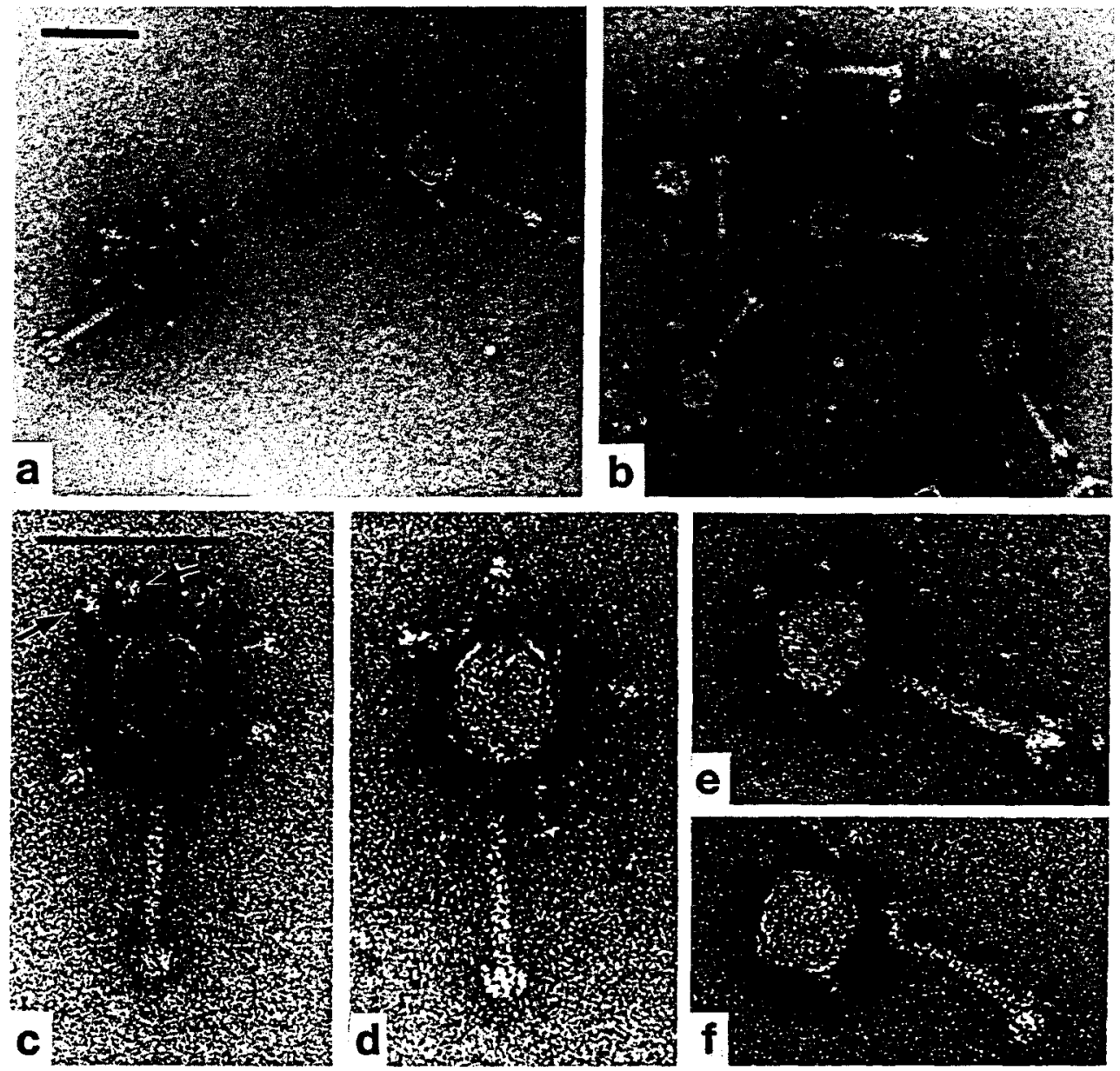

Fig. 9. Phages with usual tail appendages, negatively stained with UA. a, $c$, and $d$ : phage A762/2. $b, e$, and $f$ : phage $H 47 / 4$. Arrows in $c$ mark trilobate end structure of antennae. Bar represents $100 \mathrm{~nm}$

considerably longer tail. Additionally, this phage has an arrangement of large globular (?) bodies at the tip of its tail, closely resembling configurations found in the phages reported above (p. 398).

\section{Phages with short non-contractile tail (Bradley group C)}

All phage strains assigned to this group have an isometric head of hexagonal contour.

\section{Phages with no antennae on the head}

In the six members of this group, two different types of tail structure were found. Strains A45/1 (Fig. 11c) and A63/1 (Fig. 11a, b) closely resemble the coliphages T3 and 

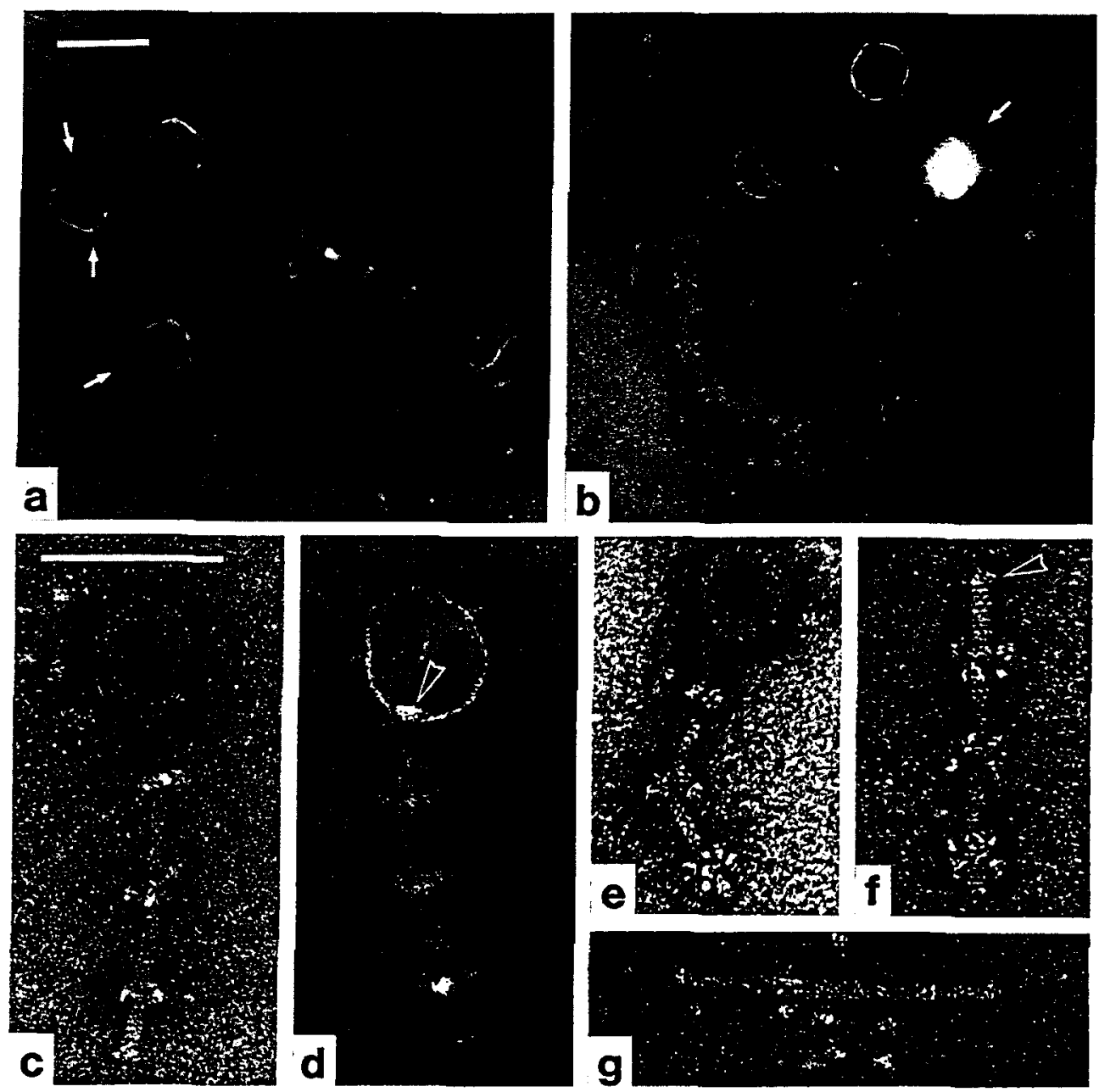

Fig. 10. Phage A660/3, the only representative of the group, negatively stained with phosphotungstic acid $(a, b)$ or UA $(c-g)$. Arrows in $a$ and $b$ indicate some of the faintly visible antennae, arrowheads in $d$ and $f$ mark adaptor protein linking head and tail. Bar represents $100 \mathrm{~nm}$

T7 in having a wedge-shaped tail with usually no structural details discernible. In a few particles, however, tiny structures at the base of the tail were observed (Fig. 11b, c). The remaining bacteriophage strains of this group appear to be similar to the Salmonella phage P22. Their tail is more complex and consists of claw-like pins the number of which is probably six (Fig. 11d, e). The existence of a short central tube cannot be ruled out. 

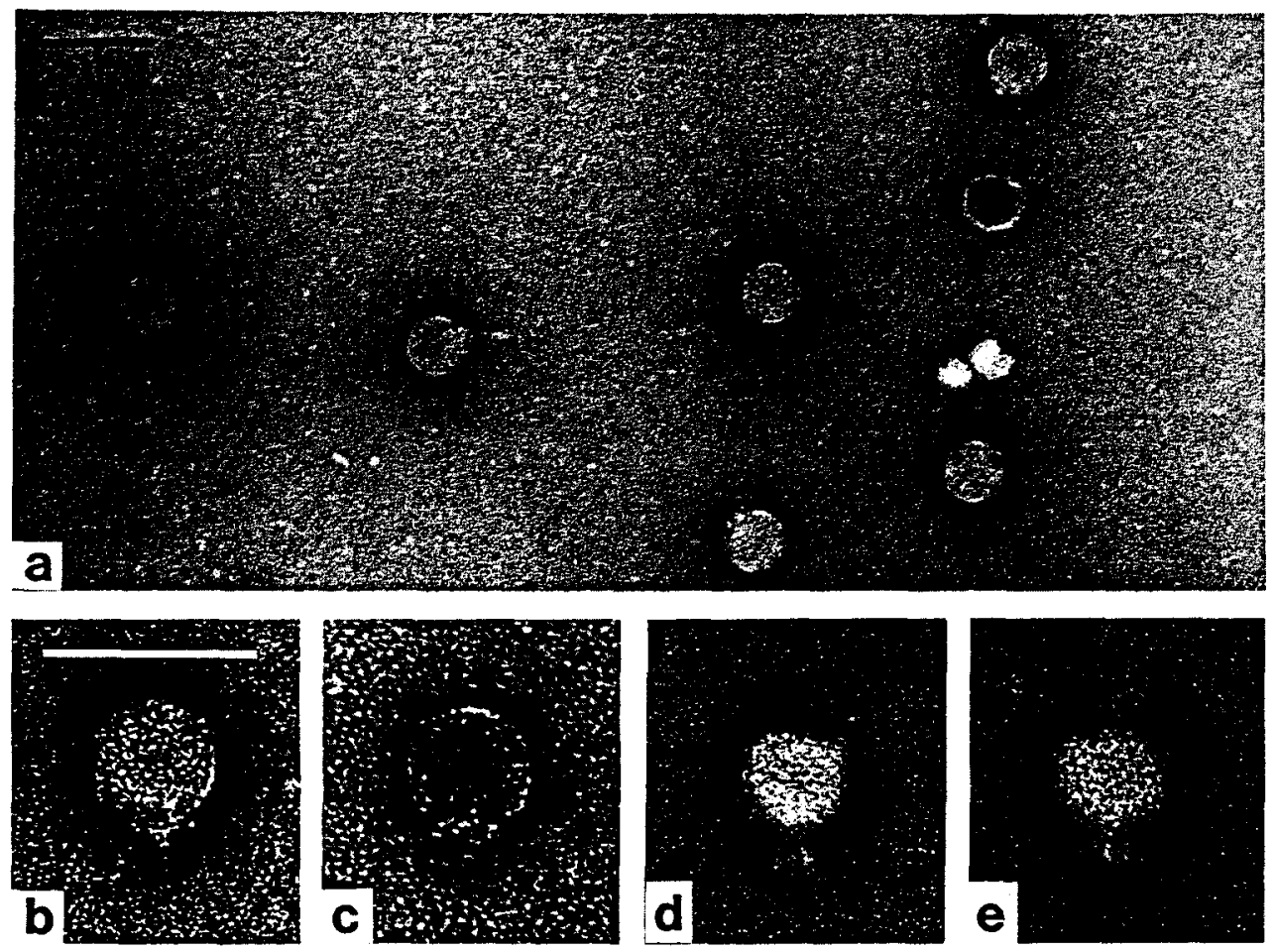

Fig. 11. Phages with no antennae, negatively stained with UA. $a, b$ : phage A63/1. c: phage A45/1. d, e: phage $\mathrm{H} 45 / 1$. Bar represents $100 \mathrm{~nm}$

\section{Phages with antennae on the head}

Phages with no unusual appendages on the tail

The four members of this group also show some remarkable differences in the morphology of their tail structure apart from other traits.

Strain H17/1 (Fig. 12) has short knob-like antennae (Fig. 12b, $\mathrm{i}-\mathrm{n}$ ) and a very short tail (Fig. 12b-d, arrowhead). The antennae are attached to the vertices and consist of several subunits (Fig. 12i, arrow, and $\mathrm{m}$ ). The small disk-like structure with hexagonal outline (Fig. 12c, d, arrow; 12e at higher magnification), frequently found in $H 117 / 1$ preparations, are assumed to be part of the tail structure, possibly a 'base plate'. The position of this structure within the set-up of the virion is unknown. Depending on the degree of penetration by stain (Fig. 12d, f) or in ultrathin sections (Fig. 12g, h) a multilayered structure of the head may become discernible. From these observations, the existence of at least two distinct shells enveloping a core is deduced. Accordingly, the particles shown in Figure 120 are regarded as virions, the outer shell of which is disintegrating, with the inner shell and core discernible in the particle indicated by arrow. In Figure 12p, the arrowhead points to a particle with the outer shell disintegrated, and the arrow indicates a structure assumed to be the core of a virion with both the outer shells removed. Its almost hexagonal contour may be caused by a third (innermost) shell 

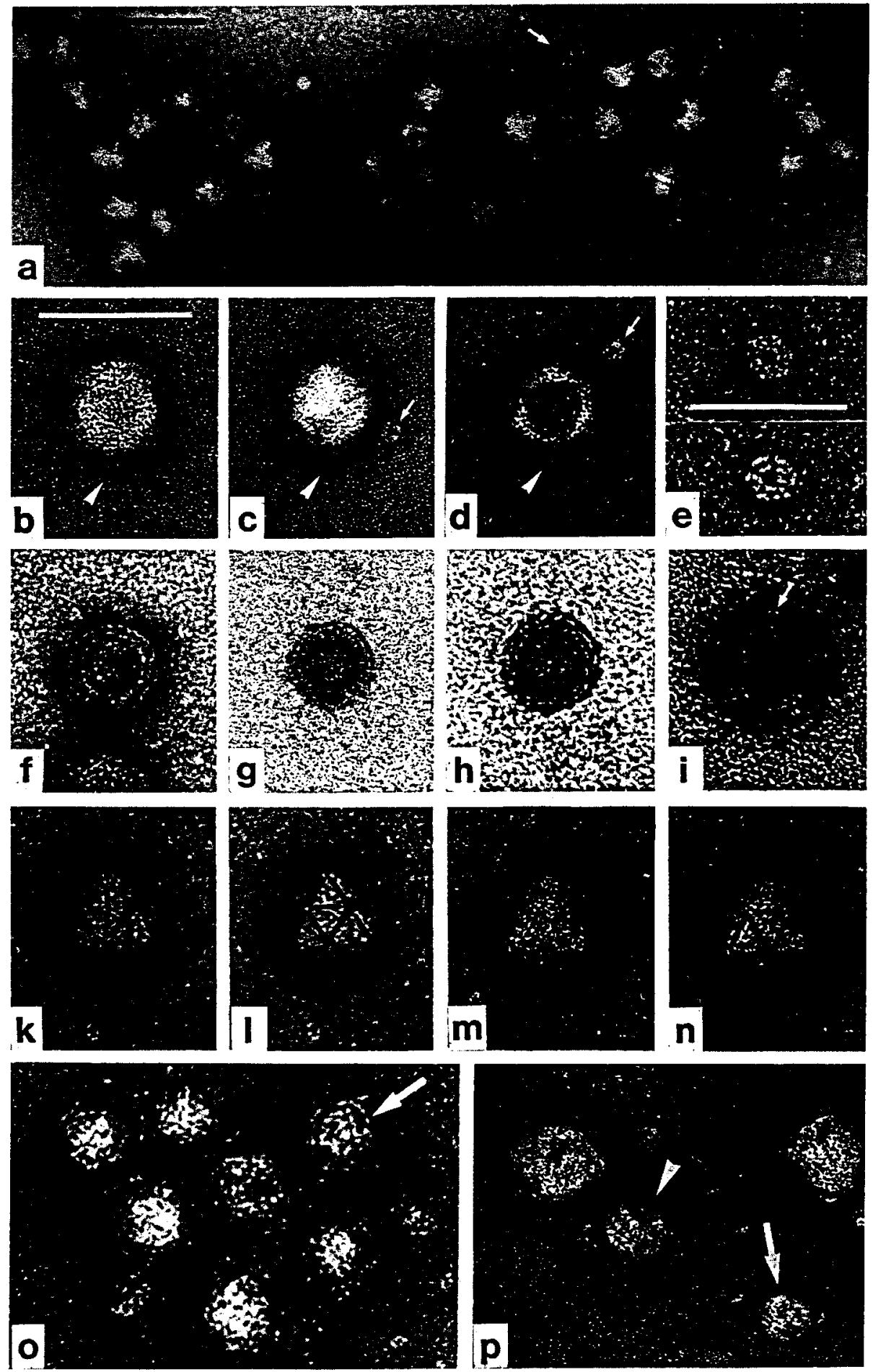
still in place, the existence of which is possibly indicated in ultrathin sections (Fig. 12g, h).

Owing to one-sided staining of phage heads, structures on their surface may become visible. In Figures $12 \mathrm{k}$ and $12 \mathrm{~m}$, two virions are presented with antennae discernible both on their surface and periphery. Lines drawn between the antennae extending from the vertices (Fig. 12l, n) disclose the icosahedral structure of the $\mathrm{H} 17 / 1$ virions. In Figure 12i, a virion is shown in a position revealing the fivefold symmetry of an icosahedron. There are only a few more viruses known to show their icosahedral symmetry in such clarity (e.g. Adeno virus).

The other three members of this group (strains A88/1, A93/1, and A1482/1) also have knob-like antennae which appear to be somewhat larger than those of strain $H 17 / 1$ (Fig. 13a-d). In strain A1482/1, these knobs were observed to consist of several subunits (Fig. 13d). The tail of these phages is considerably larger than that of $H 17 / 1$. Its structure is similar to that of phages with no antennae (Fig. 11d, e) in that it is mainly composed of claw-like pins. The head-on view of a virion of strain A93/1 (Fig. 13e) reveals that six pins surround a central tube.

\section{Phages with unusual tail fibres}

The virions of the nine phage strains comprising this group have basically a morphology similar to the three strains described in the last paragraph. Their isometric head of hexagonal outline has a short tail with probably six claw-like pins or spikes. Their antennae, extending from the vertices, are either knob-like structures (Fig. 14b) or short stems, or filaments tipped with small bodies (Fig. 14f, $g$, and i). The structure of the antennae and possible differences between the nine strains regarding this type of appendage remain dubious.

The feature which sets these phage strains apart from the others belonging to group $\mathrm{C}$ of Bradley's, however, is the occurrence of tail fibres bearing trilobate bodies on the distal end. The length of the fibres varies slightly in the different strains (compare in Fig. 14: $b-e$ with $h$ and i). These fragile structures were most abundantly found in strain A214/2 (Fig. 14a-e). Among the several hundred of virions scrutinized, many were observed with five fibres but only very few if any with six fibres. As seen in Figure 14d, the fibres extend from a plate-like structure which may have a sixfold symmetry (inset in Fig. 14a).

Fig. 12. Phage $H 17 / 1$, negatively stained with UA ( $a-f$ and $i-p$ ) or ultrathinly sectioned $(g, h)$. $a$ : general view, black arrows mark particles with disintegrating outer shell, white arrow points to inner shell of disintegrated particle. $b-d$ : particles with presumed tail indicated by arrowheads. Arrows in $c$ and $d$ mark disk-like structures of hexagonal outline, shown at higher magnification in $e$, which may represent lost base plates. $f$ : particle penetrated by UA, revealing existence of a multi-layered envelope, $g, h$ : ultrathinly sectioned particles revealing three-layered capsid structure. $i$ : particle in position showing fivefold symmetry axis of an icosahedron, with arrow indicating antenna with subunits discernible. $k, l$ and $m, n$ : virions with one-sided staining, showing the protrusions on vertices in topview. Connections drawn between protrusions (Figs $l$ and $n$ ) demonstrate icosahedral symmetry of phage head. $o$ : particles with disintegrating outer shell, arrow marks a virion with inner shell discernible because of UA penetration. p: particles in various stages of disintegration, arrowhead indicating one with outer shell lost and arrow marking another with second shell lost, too. Its remainder has still a somewhat hexagonal outline, possibly indicating existence of a third shell. Bar represents $100 \mathrm{~nm}$ except for $e(=50 \mathrm{~nm})$ 


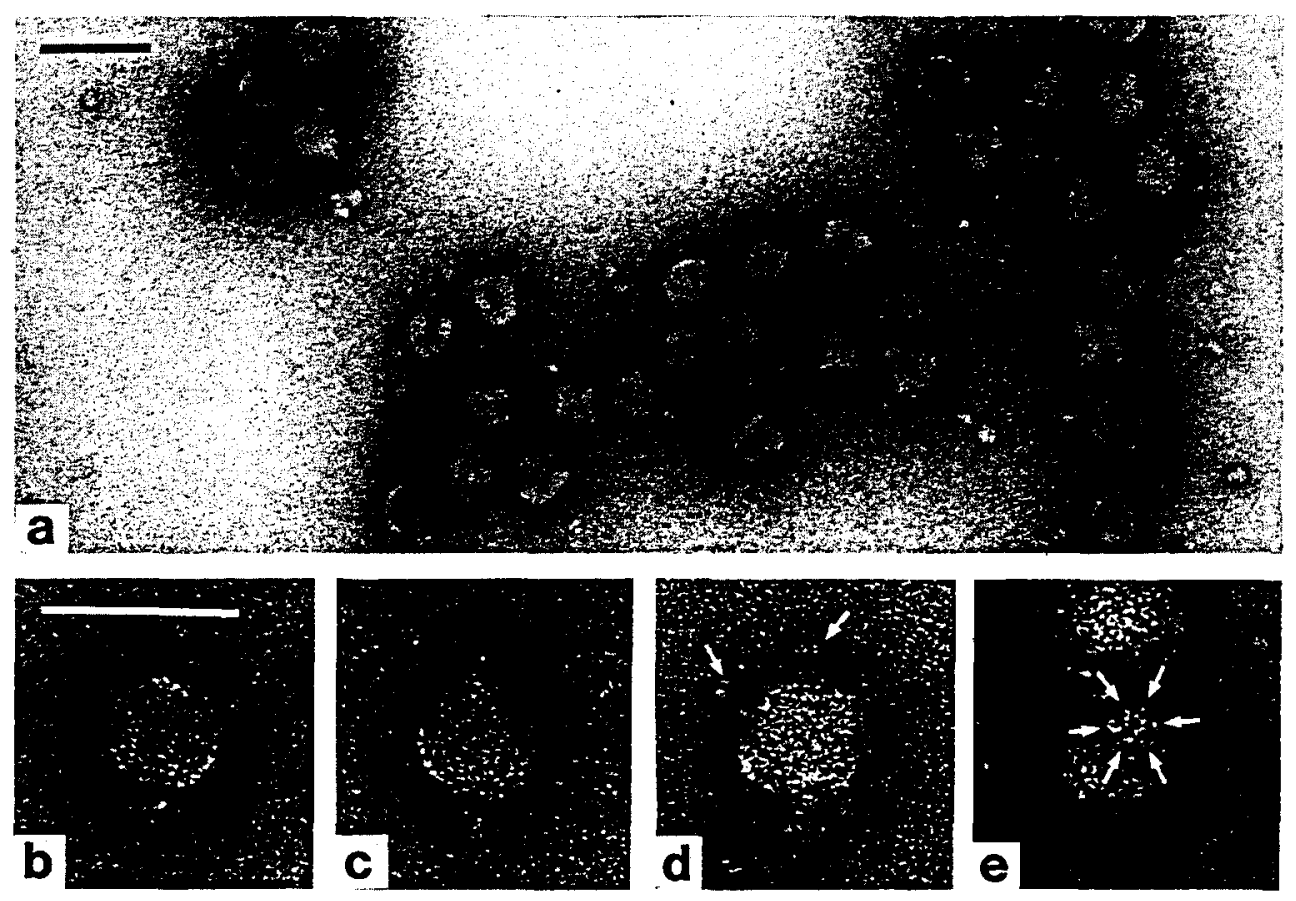

Fig. 13. Other phages with no unusual tail appendages, negatively stained with UA. a, $d$ : phage A1482/1, arrows in $d$ point to antennae with subunits discernible. $b, c$ : phage A88/1. e: phage A93/1, arrows indicating sixfold symmetry of base plate. Bar represents $100 \mathrm{~nm}$

\section{DISCUSSION}

As far as can be judged from the available information, no bacteriophages of marine or nonmarine origin have been described so far by other authors that would fit into four sections of this presentation (pp. 392/393, Figs 2 and 3, p. 397, Fig. 5, p. 398, Fig. 7, p. 401, Fig. 9). In addition, of two somewhat similar phages the single strain (A660/3) described on p. 402 (Fig. 10) is the only one derived from a marine environment. These sections comprise phage strains characterized by conspicuous appendages such as long antennae or tail fibres. It may be merely accidental that phages of corresponding morphology have not been isolated up till now. As will be discussed below, there are indications of the existence of morphologically different bacteriophage populations restricted to certain areas in the northern Atlantic. Since the majority of marine phages, the morphology of which was reported by other authors, were isolated from samples taken in the Pacific, the possibility exists that phages fitting into the sections mentioned above were missed due to geographic separation. However, there is also a possibility that appendages characteristic of the phages included in these sections, being of rather fragile structure, were lost during preparation due to the methods employed by other investigators.

There is one example to be pointed out in this context. Hidaka \& Fujimura (1971) who used $1 \%$ ammonium acetate solution as medium for pelleted phage particles and 

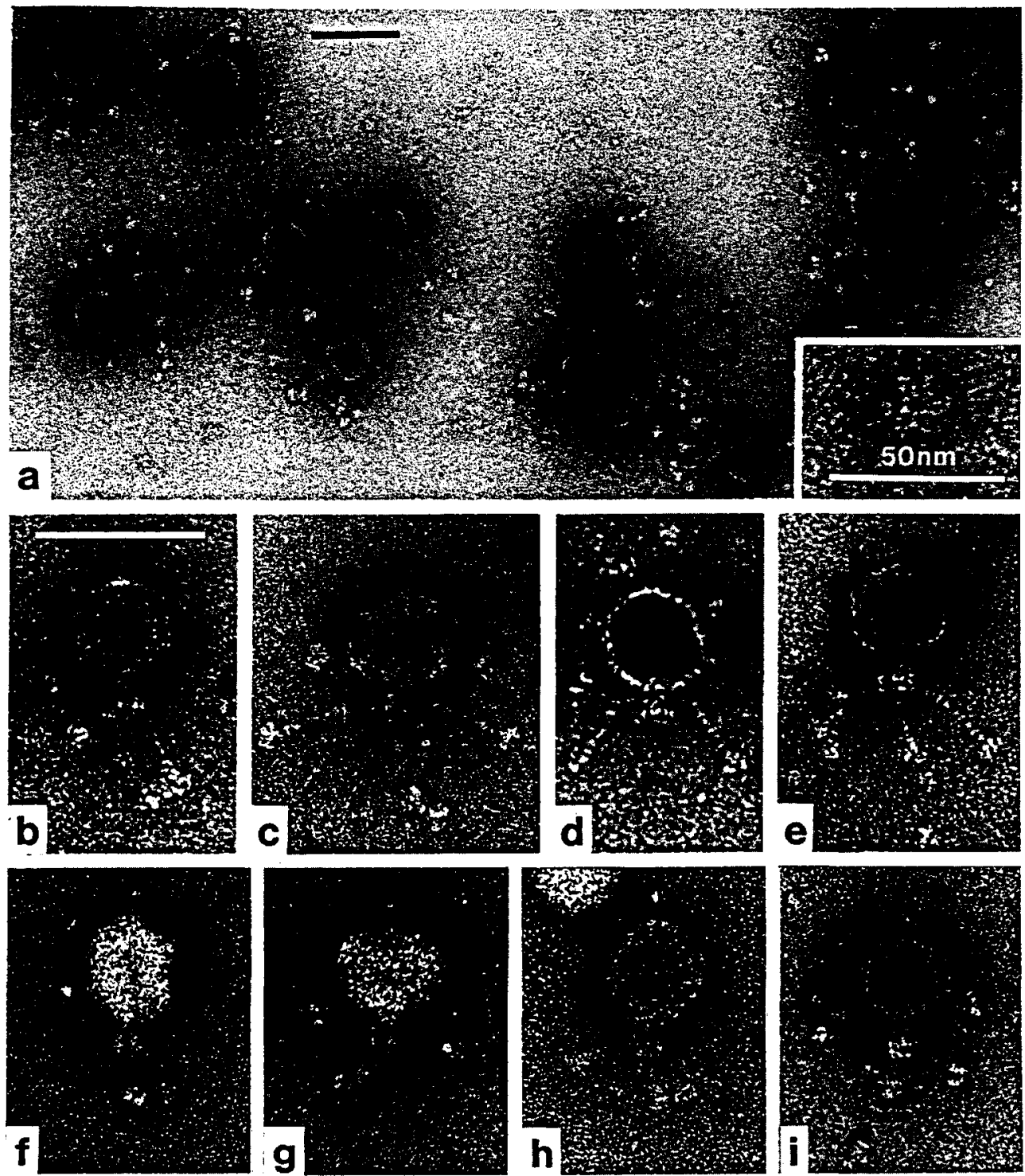

Fig. 14. Phages with unusual tail fibres, negatively stained with UA. a-e: phage A214/2. Inset in a: disk-like structure, possibly representing a base plate as indicated by its sixfold symmetry. $f, g$ : phage A1464/3. h: phage A1474/1, i: phage A1288/1. Bar represents $100 \mathrm{~nm}$ except for the insert in $a$

phosphotungstic acid as stain, isolated one phage strain (OXN-100P) which fits the description given on p. 407 of this investigation. The two particles presented by the authors, however, bear only one and three appendages, respectively, that correspond structurally to those observed in our phages, i.e. long tail fibres with trilobate structures at the tip. Phage 2ZT-33P of Hidaka (1973) may also belong to this group, but in this case 
only one of two virions demonstrated faintly shows one (or two?) such appendages. According to our experience with 9 strains, the appendages in question are of considerable, though varying, stability. The different findings obtained with similar phages may therefore be due to the variant methods employed, although they may be caused also by differences among the phage strains.

Phages morphologically similar to those ascribed to the remaining sections of this investigation were reported by several authors. Those which would fit the descriptions given on pp. 388 (Fig. 1), 398 (Fig. 6) and 403, 404 (Fig. 11) were most commonly encountered. Three strains fitting the descriptions presented on pp. 396 (Fig. 4) and 407 (Fig. 13) were isolated by Hidaka \& Fujimura (1971). Zachary (1974) reported a phage similar to our strain $\mathrm{H} 23 / 1$ (p. 401 [Fig 8]) but ascribed it to the Bradley group $A$, indicating the presence of a contractile sheath. Two non-marine phages of similar appearance, infective for Rhizobium melilotis, are described by Fraenkel-Conrat (1986) as "probably Myoviridae", also pointing to the (probable) existence of a contractile sheath. In our strain H23/1, no indication of such a structure was observed. Phages isolated by Hidaka (1973) and Hidaka \& Fujimura (1971) which very probably could be ascribed to the group presented on p. 407 (below) have already been mentioned.

On the other hand, among the phages included in this investigation several morphological types reported by other authors were not encountered. Hidaka \& Fujimura (1971) isolated phages with non-contractile tails, the length of which was about or more than three times that of the head. Koga \& Kawata (1981) reported a phage strain with long non-contractile tail and a head covered with knob-like projections. A similar looking strain was isolated by Keynan et al. (1974). Hidaka \& Fujimura (1971) and Zachary (1974) reported one phage strain each belonging to group A of Bradley's, with a head about twice as long as wide, and another group A phage isolated by Zachary (1974) measured, extraordinarily, $480 \mathrm{~nm}$ in length. The last mentioned author also observed phage particles possibly representing members of the Bradley group D or $\mathrm{E}$.

For reasons of no bearing on the topic of this paper, phage strain H17/1 (p. 405) is the most attentively investigated one among the 75 strains included in the present study. It is conspicuously sensitive to temperatures below $4{ }^{\circ} \mathrm{C}$ and above $25^{\circ} \mathrm{C}$ and extremely sensitive to chloroform. The stability of phage stocks prepared with various media and by different methods is embarrassingly low. Finally, it is a semi-temperate bacteriophage (Moebus, unpublished data). In some of these characters as well as in its morphology, H17/1 closely resembles the phage strain O7T-12P isolated by Hidaka (1973) and investigated in greater detail by Hidaka \& Shirahama (1974). Another similar phage is strain O6N-58P of Hidaka \& Fujimura (1971), further studied by Hidaka \& Ichida (1976). The latter authors assumed that, because of its extreme sensitivity to chloroform, this strain may be another lipid-containing marine bacteriophage. Strain O6N-58P is the only RNA-containing marine phage reported so far.

Strain H17/1 also resembles phage PM2 of Espejo \& Canelo (1968) which was the first lipid-containing bacteriophage isolated and, therefore, attracted the attention of many authors (for review see Mindich, 1978). As reported by Espejo \& Canelo (1968), strain PM2 depends on low temperature for survival and reproduction and is highly sensitive to organic solvents. According to Silbert et al. (1969) PM2 does not show any tail structure. These authors observed small projections only on the vertices of the virions, which with some particles were seen in a splayed configuration. As the tail structure of 
H17/1 morphologically remains dubious it may in reality be similar to the splayed projections of PM2.

Of far more interest than the doubtful tail structure is the set-up of the H17/1 capsid which very closely resembles findings reported for PM2. The early observation of a double-layered coat discernible in negatively stained particles (Espejo \& Canelo, 1968) were confirmed by Silbert et al. (1969) who found PM2 to be built of an outer and an inner shell surrounding a core. Further investigations on PM2 performed by Hinnen et al. (1974) and Schäfer et al. (1974) resulted in a model of this phage which, besides other details, includes an outer and an inner shell consisting of protein II and III, respectively, and a phospholipid bilayer in between. As demonstrated in Figure $12(a, d, f-h, o, p)$, our observations on $\mathrm{H} 17 / 1$ indicate the existence of at least two shells enveloping a core. The three layers surrounding the core, observed in ultrathin sections (Fig. 12g, h), are possibly in agreement with the model of Schäfer et al. (1974) developed for phage M2. Together with the extreme sensitivity to chloroform, our findings point out the possibility that $\mathrm{H} 17 / 1$ is another lipid-containing phage of marine origin. They also may serve as a clue for understanding the rapid loss of infectivity of H17/1 during storage under any of the conditions tested so far.

The bacteriophages described in this paper were isolated from water sampled in various regions of the northern Atlantic (A-series) or near Helgoland ( $\mathrm{H}$-series). About the same degree of morphological diversity, on the one hand, and morphologically similar types, on the other hand, were found among the bacteriophages derived from both the Atlantic and the North Sea. However, there is one conspicuous difference between the phage strains of both series concerning the occurrence of unusual appendages of some length (knob-like antennae excluded). While of the $20 \mathrm{H}$-series phages investigated only 3-4 strains (H7/2 and H84/1, p. 394 [below]; H36/1 and, possibly, H54/ 1 , p. 401 [above]) were observed to have such structures, the respective number with 55 A-series strains, however, is 24.

At present, one can only speculate on the functions of appendages such as long antennae or tail fibres. One possible interpretation could be that such structures may help to secure adsorption of a phage particle onto the host cell, and another would imply that, by such 'far reaching' appendages, the chances of a virion meeting a host cell increase, provided that the appendages themselves are suited to recognize host cells among all the particulate matter a virion may encounter. Considering the much greater rareness of cultivatable bacteria in the Atlantic as compared with the North Sea, probably reflecting similar differences between both ecosystems regarding the frequency of suitable host bacteria, the high incidence of long appendages among the bacteriophages from the Atlantic seems, in the light of the above hypotheses, intelligible.

As reported by Moebus \& Nattkemper (1981) and Moebus (1983), considerable differences with regard to phage sensitivity patterns were found between bacterial isolates collected east or west of the Azores, respectively. Considering the present results in view of the earlier ones, the following observations were made with the A-series phages investigated by electron microscopy (phage mutant A1536/1-1, see Table 2, excluded from consideration):

Among the 19 strains briefly described on p. 388 (Fig. 1), their morphology roughly resembling that of the $\mathrm{T}$-even coliphages, only 4 were derived from samples taken west of the Azores ("western" strains, strain numbers from 183 to 1242). These 4 strains were 
the only ones of this group able to lyse "western" bacteria, and 3 of them lysed only "western" bacteria. The 15 "eastern", phage strains lysed "eastern" bacteria only. With all 19 strains very narrow host ranges were observed, encompassing between 1 and 6 bacterial strains among the 733 A-series bacteria tested (Moebus \& Nattkemper, 1981; Moebus, 1983, and unpublished data).

The 7 morphologically inseparable phage strains described on p. 392-393 (Fig. 2) were derived from the westernmost part of the Atlantic sampled (west of $45^{\circ} \mathrm{W}$ ). In these phages, extensively overlapping host ranges were found covering $60 \mathrm{~A}$-series bacteria representing 42 different phage sensitivity patterns. The phages lysed 50 "western" bacteria collected in the whole range of the "western" Atlantic sampled as well as 10 "eastern" bacteria. The latter were isolated from 3 water samples taken between $10^{\circ} \mathrm{W}$ and $15^{\circ} \mathrm{W}$, i.e. far away from the sampling sites of these bacteriophages and within a relatively narrow range. These "eastern" bacteria were also lysed by phages A1482/1 and A1464/3 which both are "eastern" phages belonging to the Bradley group C (see Table 2). Phage strain A720/1 (Fig. 2f-h), set apart from the aforementioned 7 strains by shorter tail fibres, lysed only the bacterial strain used for its isolation (Moebus \& Nattkemper, 1981; Moebus, 1983).

Similar observations were made regarding phage strains described on p. 398 (below) (Fig. 7). The phages A275/1, A660/1, and A882/1 are morphologically inseparable which corresponds with widely overlapping host ranges. By these phages 10 "western" bacterial isolates were lysed, but in 14 "eastern" bacteria lysis or inhibition was observed, both with about the same frequency. In strain A701/1, differing from the 3 aforementioned ones by a slightly shorter tail, no similarity in host range was detected. Instead, its host range overlaps with that of strain A289/1 (Moebus \& Nattkemper, 1981; Moebus, 1983 , and unpublished data), which is assigned to the same group but differs from the 4 strains mentioned before by the apparent lack of unusual appendages of the tail and a head less easily destroyed during preparation for electron microscopy. (It should be pointed out here that the assignment of all phages to the various groups is based exclusively on morphological traits.)

Observations made with the 15 phage strains found to belong to Bradley's group $\mathrm{C}$ are quite similar to those discussed above regarding the phage strains described on p. 388. Of these strains 12 are "eastern" ones, and of the 3 "western" strains two (A214/2 and A216/2) were isolated within the range of the Azores. Except for the 3 "western" phages, exclusively lysing their respective "western" host, these phages lysed only "eastern" bacteria, and almost no similarity in host ranges was found.

Moebus \& Nattkemper (1981) concluded from their findings concerning the phage sensitivity patterns of Atlantic Ocean bacteria that considerable differences exist between "eastern" and "western" microbial populations. They also pointed out that such differences may even exist between less widely separated populations. The results of this investigation strongly support their view. As indicated by the observations discussed above, some of the groups of morphologically different bacteriophages comprise strains derived from distinct areas in the Atlantic. In one case, it was a remarkably small one: five of the 7 morphologically identical phages described on p. 392-393 (Fig. 2) were isolated from samples taken in the Sargasso Sea, with a sixth strain found near the Bermudas.

One might argue that the number of phage strains investigated is too low to support such far-reaching conclusions, and that our observations may be biased by the fact that 
only phage strains were investigated which, by using standard methods, yielded hightitre phage suspensions. The latter point may certainly be important in regard to the number of different morphological types observed, but concerning the assumed geographic separation of structurally different bacteriophage types it seems of little bearing. Firstly, $50 \%$ of the phage strains which met with the precondition mentioned were investigated. Secondly, the phages were selected according to the assignment of their 'original' host (i.e. bacteria used for isolation of respective phage) to 10 groups comprising between 2 and 128 bacterial isolates, 9 of which combined bacteria related by phage sensitivity patterns. In the selection of phages from these groups, established phage sensitivity patterns were disregarded, i.e. selection of phages with highly similar host ranges occurred in a random fashion. Thirdly, wherever possible, phages of different geographic origin within these groups were selected. Altogether, 23 "western" and 31 "eastern" phage strains were investigated. For these reasons, our observation of "eastern" and "western" morphological types among the investigated bacteriophages is not regarded as severely biased but as substantially reflecting the natural differences between bacteriophage populations present east and west of the Azores, respectively, in the northern Atlantic.

As for the $\mathrm{H}$-series bacteriophages investigated, widely overlapping host ranges were observed with strains H7/2, H54/1, and H84/1 only (Moebus \& Nattkemper, 1981). Both the first and last mentioned strains were found to be very similar morphologically (p. 394 [Fig. 3]), but strain H54/1 (p. 401 [above]) is completely different. In these 3 strains the aforementioned authors also observed lytic or inhibitory activity on quite a number of A-series bacteria, which were almost all "western" isolates and, except one, insensitive to A-series bacteriophages. All but one of the other $\mathrm{H}$-series phages used in this investigation were found to lyse only the respective original host. The exceptional strain (H15/1) lysed one additional bacterial isolate (Moebus \& Nattkemper, 1981).

Acknowledgements. The authors are grateful for the reliable technical assistance of Mrs. A. Kleih, Miss R. Braun, Mr. J. Berger and Mrs. I. Zimmermann. Thanks are also due for excellent photographic work to Mrs. R. Groemke-Lutz, and Miss B. Sailer, as well as to Miss D. Eder for her support in preparing Table 1.

\section{LITERATURE CITED}

Bradley, D. E., 1966. The fluorescent staining of bacteriophage nucleic acid. - J. gen. Microbiol. 44, 383-391.

Bradley, D. E., 1967. Ultrastructure of bacteriophages and bacteriocins. - Bact. Rev. 31, $230-314$.

Espejo, R. T. \& Canelo, E. S., 1968. Properties of bacteriophage PM2: A lipid-containing bacterial virus. - Virology 34, 738-747.

Fraenkel-Conrat, H., 1986. The viruses: catalogue, characterization, and classification. Plenum Press, New York, $268 \mathrm{pp}$.

Frank, H. \& Day, L. A., 1970. Electron microscopic observations on fd bacteriophage, its alkali denaturation products and its DNA. - Virology 42, 144-154.

Hidaka, T., 1973. Characterization of marine bacteriophages newly isolated. - Mem. Fac. Fish. Kagoshima Univ. 22, 47-61.

Hidaka, T. \& Fujimura, T., 1971. A morphological study of marine bacteriophages. - Mem. Fac. Fish. Kagoshima Univ. 20, 141-154.

Hidaka, T. \& Ichida, K., 1976. Properties of a marine RNA-containing bacteriophage. - Mem. Fac. Fish. Kagoshima Univ. 25, 77-89.

Hidaka, T. \& Shirahama, T., 1974. Preliminary characteristics of a temperate phage system isolated from marine mud. - Mem. Fac. Fish. Kagoshima Univ. 23, 137-148. 
Hidaka, T. \& Tokushige, A., 1978. Isolation and characterization of Vibrio parahaemolyticus bacteriophages in sea water. - Mem. Fac. Fish. Kagoshima Univ. 27, 79-90.

Hinnen, R., Schäfer, R. \& Franklin, R. M., 1974. Structure and synthesis of a lipid-containing bacteriophage. Preparation of virus and localization of the structural proteins. - Eur. J. Biochem. $50,1-14$.

Johnson, R. M., 1968. Characteristics of a marine Vibrio-bacteriophage system. - J. Ariz. Acad. Sci. $5,28-33$.

Kakimoto, D. \& Nagatomi, H., 1972. Study of bacteriophages in Kinko Bay. - Bull. Jap. Soc. scient. Fish. 38, 271-278.

Keynan, A., Nealson, K., Sideropoulos, H. \& Hastings, J. W., 1974. Marine transducing bacteriophage attacking a luminous bacterium. - J. Virol. 14, 333-340.

Koga, T. \& Kawata, T., 1981. Structure of a novel bacteriophage VP3 for Vibrio parahaemolyticus. Microbiol. Immunol. 25, 737-740.

Koga, T., Toyoshima, S. \& Kawata, T, 1982. Morphological varieties and host range of Vibrio parahaemolyticus bacteriophages isolated from seawater. - Appl. envir. Microbiol. 44, 466-470.

Mindich, L., 1978. Bacteriophages that contain lipid. In: Comprehensive virology. Ed. by $\mathrm{H}$. Fraenkel-Conrat \& R. R. Wagner. Plenum Press, New York, 12, 271-335.

Moebus, K., 1980. A method for the detection of bacteriophages from ocean water. - Helgoländer Meeresunters. 34, 1-14.

Moebus, K., 1983. Lytic and inhibition responses to bacteriophages among marine bacteria, with special reference to the origin of phage-host systems. - Helgoländer Meeresunters. 36, 375-391.

Moebus, K. \& Nattkemper, H., 1981. Bacteriophage sensitivity patterns among bacteria isolated from marine waters. - Helgoländer Meeresunters. 34, 375-385. .

Moebus, K. \& Nattkemper, H., 1983. Taxonomic investigations of bacteriophage sensitive bacteria isolated from marine waters. - Helgoländer Meeresunters. 36, 357-373.

Nakamura, H., Kakimoto, D., Swafford, J. \& Johnson, R., 1978. Studies on the characteristics of the bacteriophages of Vibrio alginolyticus B-1 isolated from Kinko Bay. - Mem. Fac. Fish. Kagoshima Univ. 27, 59-64.

Ritchie, A. E., Robinson, I. M. \& Allison, M. J., 1970. Rumen bacteriophage: survey of morphological types. In: Septième Congrès International de Microscopie Electronique, Grenoble. Soc. Franc. Micr. Electron., Paris, 333-334.

Schäfer, R., Hinnen, R. \& Franklin, R. M., 1974. Structure and synthesis of a lipid-containing bacteriophage. Properties of the structural proteins and distribution of the phospholipid. - Eur. J. Biochem. 50, 15-27.

Sieburth, J. McN., 1979. Sea microbes. Oxford Univ. Press, New York, 491 pp.

Silbert, J. A., Salditt, M. \& Franklin, R. M., 1969. Structure and synthesis of a lipid-containing bacteriophage. III. Purification of bacteriophage PM2 and some structural studies on the virion. Virology 39, 666-681.

Sklarow, S. S., Colwell, R. R., Chapman, G. B. \& Zane, S. F., 1973. Characteristics of a Vibrio parahaemolyticus bacteriophage isolated from Atlantic coast sediment. - Can. J. Microbiol. 19, 1519-1520.

Smith, L. S. \& Krueger, A. P., 1954. Characteristics of a new Vibrio-bacteriophage system. - J. gen. Physiol. 38, 161-168.

Spencer, R., 1960. Indigenous marine bacteriophages. - J. Bact. 79, 614.

Stevenson, J. H. \& Albright, L. J., 1972. Isolation and partial characterization of a marine bacteriophage. - Z. allg. Mikrobiol. 12, 599-603.

Torrella, F. \& Morita, R. Y., 1979. Evidence by electron micrographs for a high incidence of bacteriophage particles in the waters of Yaquina Bay, Oregon: ecological and taxonomical implications. - Appl. envir. Microbiol. 37, 774-778.

Valentine, A. F., Chen, P. K., Colwell, R. R. \& Chapman, G. B., 1966. Structure of a marine bacteriophage as revealed by the negative-staining technique. - J. Bact. 91, 819-822.

Wiebe, W. J. \& Liston, J., 1968. Isolation and characterization of a marine bacteriophage. - Mar. Biol. 1, 244-249.

Zachary, A., 1974. Isolation of bacteriophages of the marine bacterium Beneckea natriegens from coastal salt marshes. - Appl. Microbiol. 27, 980-982. 